\title{
SuperTracer: a calculator of functional supertraces for one-loop EFT matching
}

Javier Fuentes-Martín, ${ }^{a}$ Matthias König, ${ }^{b}$ Julie Pagès, ${ }^{c}$ Anders Eller Thomsen ${ }^{c, d}$ and Felix Wilsch ${ }^{c}$

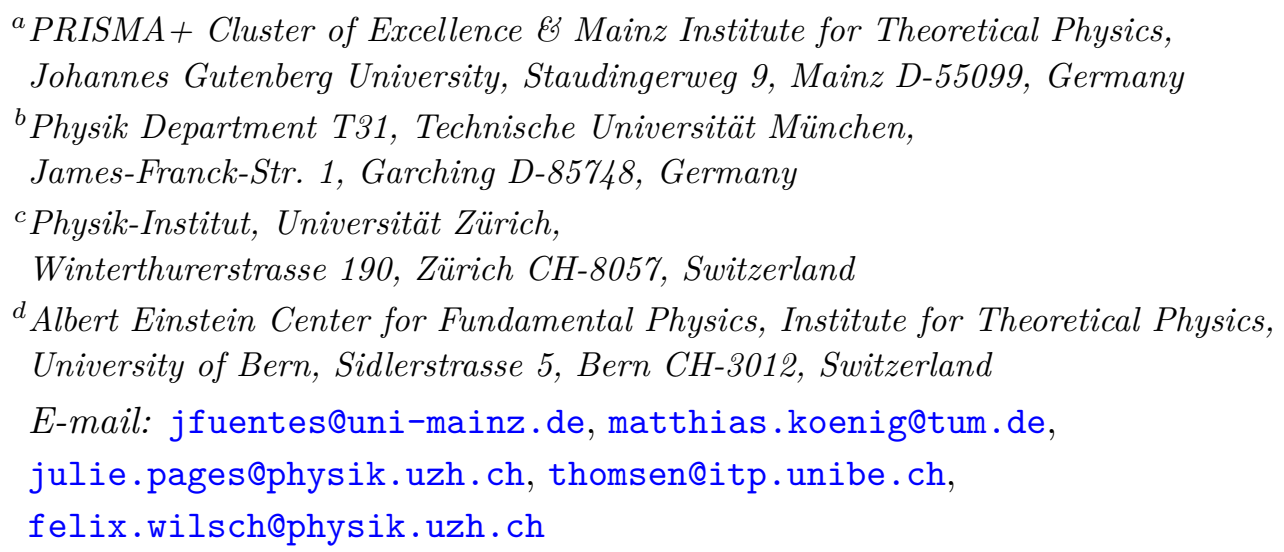

ABSTRACT: We present SuperTracer, a Mathematica package aimed at facilitating the functional matching procedure for generic UV models. This package automates the most tedious parts of one-loop functional matching computations. Namely, the determination and evaluation of all relevant supertraces, including loop integration and Dirac algebra manipulations. The current version of SuperTracer also contains a limited set of output simplifications. However, a further reduction of the output to a minimal basis using Fierz identities, integration by parts, simplification of Dirac structures, and/or light field redefinitions might still be necessary. The code and example notebooks are publicly available at $\hat{\Delta} .^{1}$

KEYwords: Effective Field Theories, Beyond Standard Model

ArXiv EPrint: 2012.08506

\footnotetext{
${ }^{1}$ https://gitlab.com/supertracer/supertracer.
} 


\section{Contents}

1 Introduction 1

2 The functional matching procedure $\quad 2$

2.1 Covariant evaluation of supertraces 5

2.2 Explicit evaluation of the relevant supertraces 6

$\begin{array}{lll}2.2 .1 & \text { Log-type supertraces } & 7\end{array}$

$\begin{array}{lll}2.2 .2 & \text { Power-type supertraces } & 8\end{array}$

2.3 Comments on the treatment of $\gamma_{5}$ in fermion supertraces 9

2.4 Summary of the functional matching method 10

3 SuperTracer in a nutshell 12

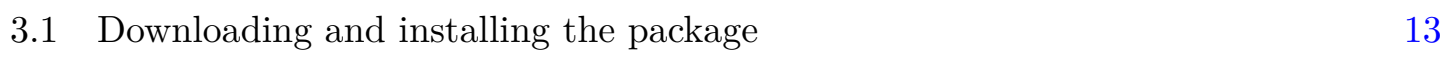

$\begin{array}{lll}3.2 & \text { SuperTracer global variables and routines } & 13\end{array}$

4 Usage examples $\quad 17$

$\begin{array}{lll}\text { 4.1 Toy model with a heavy vector-like fermion } & 17\end{array}$

$\begin{array}{lll}4.2 & S_{1} \text { scalar leptoquark } & 22\end{array}$

5 Conclusions $\quad 27$

$\begin{array}{ll}\text { A Special SuperTracer variables } & 28\end{array}$

B Diagrammatic matching for the vector-like fermion example 28

\section{Introduction}

Studying the low-energy phenomenology of a quantum field theory is an important step in most studies in the Standard Model (SM) and beyond. To this end, one constructs the corresponding effective Lagrangian by separating the relevant dynamics from different energy scales and removing the ones lying at high energies. The resulting effective field theory (EFT) can then be used to study the physics at low energies, while keeping large logarithms of the scale hierarchies under control by renormalization-group improvement. A common example of such an approach is when one studies the effects of new-physics (NP) models on flavor observables, where one separates dynamics at and above the weak scale through a series of matching steps from the dynamics at the characteristic scale of the process. In practice, one matches a given UV theory to the Standard Model Effective Field Theory (SMEFT) [1,2] which is then in turn, after renormalization group evolution (RGE) in the SMEFT [3-6], matched to the Low Energy Effective Theory (LEFT) [7-9] and evolved via the LEFT RG equations [10].

The example of flavor physics also shows the importance of performing the matching steps beyond the leading order, since a great amount of interesting observables (e.g. FCNC 
processes like rare decays and neutral meson mixing) are generated starting only at one-loop order within the SM. The process of matching NP models to an EFT to study the relevant low-energy phenomenology constitutes a repetitive and time-consuming task, calling for an automated solution. In the recent years, many tools for automated EFT calculations, specially in the context of the SMEFT, have been developed [11-25]. In particular, tools for RGE in the SMEFT and LEFT and one-loop matching of the SMEFT to the LEFT [11-13], tree-level EFT matching of generic UV models [14] (see also [26]), as well as partial one-loop EFT matching results [25, 27-29] are available. Moreover, the Matchmaker package (not yet released) will automate the diagrammatic EFT matching of generic UV models [15, 30]. However, to our knowledge, no tool for complete one-loop EFT matching is currently publicly available. We provide here a first building block in this direction by introducing SuperTracer, a Mathematica package aimed at facilitating the one-loop EFT matching of generic UV models using path integral methods.

The path integral formulation of one-loop EFT matching [27-29, 31, 32, 32-53] has clear advantages over the diagrammatic procedure. For example, one does not need to handle Feynman diagrams nor symmetry factors, and one obtains directly the complete set of EFT operators together with their matching coefficients, without requiring any prior knowledge of the EFT operator structure, symmetries, etc. All of these points and the systematic nature of the procedure render the functional approach exceptionally suited to be implemented in a computer program. As we describe in detail in section 2, at the heart of the functional one-loop matching procedure is the evaluation of functional supertraces. SuperTracer provides the full list of relevant supertraces for a given set of interactions and automates their calculation to an arbitrary order in the heavy mass expansion, limited only by the rapidly increasing complexity of the calculation at higher orders. ${ }^{1}$ Furthermore, it provides the option of inputting substitutions for the interactions in terms of fields, and applies some output simplifications. These arguably constitute the most tedious parts of one-loop functional matching computations. An important difference with other functional approaches is that the evaluation of the supertraces is performed in a manifestly covariant way by the application of the so-called Covariant Derivative Expansion (CDE) [31-33].

The structure of this paper is as follows: in section 2, we outline the functional procedure used in SuperTracer. Readers unconcerned about the theoretical details can skip to section 2.4, where we list all the steps that are needed to perform the one-loop EFT matching. Section 3 then gives a short manual of the package and its functions. In section 4 we demonstrate the usage of the package with two examples: a toy model with a heavy vector-like fermion and an $S_{1}$ scalar leptoquark extension of the SM. We conclude in section 5. Further details about SuperTracer special variables and crosschecks are given in two appendices.

\section{The functional matching procedure}

Consider a general theory $\mathcal{L}_{\mathrm{UV}}\left[\eta_{H}, \eta_{L}\right]$, whose field content can be split into heavy $\eta_{H}$ and light $\eta_{L}$ degrees of freedom, satisfying $m_{H} \gg m_{L}$. Our aim is to compute the EFT resulting

\footnotetext{
${ }^{1}$ During the completion of this project, we became aware of STrEAM [54], a package with a similar scope that is released at the same time.
} 
from integrating out the heavy degrees of freedom $\eta_{H}$ at the one-loop order. This can be done following a path integral approach for the effective action of the theory. Collecting heavy and light fields into a field multiplet of the form $\eta=\left(\eta_{H} \eta_{L}\right)^{\top},{ }^{2}$ the fields are split into background-field configurations $\hat{\eta}$ satisfying the EOMs and quantum fluctuations $\eta$, i.e. we let $\eta \rightarrow \hat{\eta}+\eta$. The effective action of the theory is then given by the path integral

$$
e^{i \Gamma_{\mathrm{UV}}[\hat{\eta}]}=\int \mathcal{D} \eta \exp \left(i \int \mathrm{d}^{d} x \mathcal{L}_{\mathrm{UV}}[\eta+\hat{\eta}]\right)
$$

Diagrammatically, the background part corresponds to tree-level lines in Feynman graphs, while lines inside loops arise from the quantum fields. Therefore, at the one-loop level, one only needs to consider terms with up to two quantum fields, since terms with more only produce contributions at higher loop orders. The Lagrangian expansion up to terms quadratic in $\eta$ reads

$$
\mathcal{L}_{\mathrm{UV}}[\hat{\eta}+\eta]=\mathcal{L}_{\mathrm{UV}}[\hat{\eta}]+\left.\frac{1}{2} \bar{\eta}_{i} \frac{\delta^{2} \mathcal{L}_{\mathrm{UV}}}{\delta \eta_{j} \delta \bar{\eta}_{i}}\right|_{\eta=\hat{\eta}} \eta_{j}+\mathcal{O}\left(\eta^{3}\right),
$$

where the bar denotes the conjugate and $\delta / \delta \eta_{i}$ is the functional derivative with respect to $\eta_{i}$. The first term $\mathcal{L}_{\mathrm{UV}}[\hat{\eta}]$ depends only on the classical field configurations and yields the tree-level effective action. At energies much lower than the mass of the heavy fields, one can perform a local expansion in inverse powers of $m_{H}$ of the heavy field EOMs to eliminate $\hat{\eta}_{H}$ in favor of the light fields. As a result, we obtain the tree-level EFT, namely we have $\mathcal{L}_{\mathrm{UV}}\left[\hat{\eta}_{L}, \hat{\eta}_{H}\left(\hat{\eta}_{L}\right)\right]=\mathcal{L}_{\mathrm{EFT}}^{(0)}$, with $\mathcal{L}_{\mathrm{EFT}}^{(0)}$ being the tree-level EFT Lagrangian. The linear term in the expansion of $\mathcal{L}_{\mathrm{UV}}$ around the background fields is, up to a total derivative, proportional to the EOMs evaluated at $\eta=\hat{\eta}$ and thus vanishes. From the quadratic piece, we identify the fluctuation operator, with the generic form

$$
\left.\mathcal{O}_{i j} \equiv \frac{\delta^{2} \mathcal{L}_{\mathrm{UV}}}{\delta \eta_{j} \delta \bar{\eta}_{i}}\right|_{\eta=\hat{\eta}}=\delta_{i j} \Delta_{i}^{-1}-X_{i j}
$$

with $\Delta_{i}^{-1}$ being the inverse propagator of $\eta_{i}$ given as ${ }^{3}$

$$
\Delta_{i}^{-1}= \begin{cases}P^{2}-M_{i}^{2} & (\text { scalar }) \\ \not P-M_{i} & (\text { fermion }) \\ -g^{\mu \nu}\left(P^{2}-M_{i}^{2}\right) & (\text { vector })\end{cases}
$$

where $P_{\mu}$ is the Hermitian covariant derivative operator $P_{\mu}(\hat{x}, \hat{q})=\hat{q}_{\mu}+g_{G} G_{\mu}^{a}(\hat{x}) T^{a}$, with $\hat{q}_{\mu}=i \partial_{\mu}$ and $P_{\mu}=i D_{\mu}$ in position space, while the $X$ terms encode the particle interactions. For practical purposes, we consider the mass operators of the light fields

\footnotetext{
${ }^{2}$ For charged degrees of freedom, the field and its complex conjugate enter as separate components in $\eta$, as we describe in more detail in 2.4.

${ }^{3}$ The vector propagator is gauge dependent, but we work exclusively in the Feynman gauge for the quantum fluctuations as a matter of practicality. This does not imply any particular choice for the classical gauge fields, which remain in the general $R_{\xi}$ gauge. See [42] for elaboration on the subject of heavy vectors in the functional method.
} 
as perturbative interaction terms in $X$ rather than part of the free Lagrangian, so their Feynman propagators appear as the ones of fully massless particles. Namely, we take $M_{i}=0$ in (2.4) for the light fields.

The one-loop effective action, thus, reads

$$
e^{i \Gamma_{\mathrm{UV}}^{(1)}}=\int \mathcal{D} \eta \exp \left(i \int \mathrm{d}^{d} x \frac{1}{2} \bar{\eta} \mathcal{O} \eta\right)
$$

This is a Gaussian path integral whose functional integration yields

$$
e^{i \Gamma_{\mathrm{UV}}^{(1)}}=(\operatorname{SDet} \mathcal{O})^{-\frac{1}{2}} \Longrightarrow \Gamma_{\mathrm{UV}}^{(1)}=\frac{i}{2} \mathrm{~S} \operatorname{Tr} \ln \mathcal{O}
$$

where the superdeterminant SDet is a generalization of the regular determinant to the case of supermatrices, i.e. matrices with Grassmann (fermionic) and ordinary (bosonic) entries. Similarly, the supertrace STr is a generalization of the trace to the case of supermatrices, carrying opposite signs for fermionic and bosonic degrees of freedom. Using the property $\mathrm{S} \operatorname{Tr} \ln (A B)=\mathrm{S} \operatorname{Tr} \ln A+\mathrm{S} \operatorname{Tr} \ln B$, valid even for non-commuting operators, and the form of $\mathcal{O}$ in $(2.3)$, we get

$$
\Gamma_{\mathrm{UV}}^{(1)}=\frac{i}{2} \mathrm{~S} \operatorname{Tr} \ln \Delta^{-1}+\frac{i}{2} \mathrm{~S} \operatorname{Tr} \ln (1-\Delta X) .
$$

This equation provides the essential building blocks for determining the one-loop EFT. However, $\Gamma_{\mathrm{UV}}$ contains all possible loop contributions, including those that would correspond to one-loop matrix elements with the tree-level EFT Lagrangian. A crucial simplification takes place by splitting $\Gamma_{\mathrm{UV}}^{(1)}$ into hard- and soft-momentum regions using the so-called method of "expansion by regions" [55, 56],

$$
\Gamma_{\mathrm{UV}}^{(1)}=\left.\Gamma_{\mathrm{UV}}^{(1)}\right|_{\text {hard }}+\left.\Gamma_{\mathrm{UV}}^{(1)}\right|_{\text {soft }},
$$

and identifying the one-loop EFT Lagrangian with the hard part of the effective action of the UV theory $[48,49]$ :

$$
\left.\Gamma_{\mathrm{UV}}^{(1)}\right|_{\text {hard }}=\int \mathrm{d}^{d} x \mathcal{L}_{\mathrm{EFT}}^{(1)} .
$$

More precisely, contributions from the hard region directly correspond to those encoded in the short-distance EFT Wilson coefficients (WCs) in $\mathcal{L}_{\mathrm{EFT}}^{(1)}$, while contributions from the soft region are the same as those from the long-distance EFT matrix elements with $\mathcal{L}_{\mathrm{EFT}}^{(0)}$. The loops containing heavy particles yield contributions from the region of hard loop momenta $p \sim m_{H}$, and from the soft momentum region, $p \sim q_{i}, m_{L}$ with $q_{i}$ being any light-particle external momenta satisfying $q_{i} \ll m_{H}$. On the other hand, loops of light particles receive contributions only from the soft momentum region. The method of expansion by regions states that the contribution of each region is obtained in dimensional regularization by expanding the loop integrand into a Taylor series in the parameters that are small there and then integrating every region over the full $d$-dimensional space of loop momenta. This statement holds up to a mismatch of divergences. Identifying the hard region with the WCs would render them infrared divergent. The mismatch is resolved once 
one also includes the hard region of the EFT amplitudes, which are all proportional to the scaleless integral

$$
\int \frac{\mathrm{d}^{d} p}{(2 \pi)^{d}} \frac{1}{p^{4}} \propto \frac{i}{16 \pi^{2}}\left(\frac{1}{\epsilon_{\mathrm{UV}}}-\frac{1}{\epsilon_{\mathrm{IR}}}\right)
$$

and have to be subtracted from the hard part, exchanging all IR divergences with UV ones. In practice, one simply does not differentiate between $\epsilon_{\mathrm{UV}}$ and $\epsilon_{\mathrm{IR}}$ and skips this last step. The trade-off is that it becomes less transparent whether the scale dependences in the matching coefficients are related to the renormalization of the UV theory or the EFT, unless one explicitly computes the counterterms of the UV theory.

Since $\Delta X \sim m_{H}^{-1}$ in the hard region, ${ }^{4}$ we can Taylor expand the second logarithm in (2.7) yielding the master formula for one-loop EFT matching [53]:

$$
\int \mathrm{d}^{d} x \mathcal{L}_{\mathrm{EFT}}^{(1)}=\left.\frac{i}{2} \mathrm{~S} \operatorname{Tr} \ln \Delta^{-1}\right|_{\text {hard }}-\left.\frac{i}{2} \sum_{n=1}^{\infty} \frac{1}{n} \mathrm{~S} \operatorname{Tr}\left[(\Delta X)^{n}\right]\right|_{\text {hard }} .
$$

This formula provides the EFT Lagrangian in terms of two types of terms: log-type and power-type supertraces. As can be seen, the log-type supertrace only depends on the heavy particle propagators, ${ }^{5}$ and is therefore universal. Namely, it only depends on the heavy particles present in the theory, but not on their interactions. On the other hand, the powertype terms depend on the particle interactions, both heavy and light, encoded in $X$. Since, as we mentioned before, $\Delta X$ is at most of $\mathcal{O}\left(m_{H}^{-1}\right)$ in the hard momentum expansion, this provides a natural truncation of the series in terms of the EFT expansion in inverse powers of $m_{H}$.

\subsection{Covariant evaluation of supertraces}

The operators appearing in the functional supertraces needed for one-loop matching are of the form $Q\left(P_{\mu}, U_{k}(\hat{x})\right)$, having a well-defined rational expansion in its arguments, where $P_{\mu}$ is the covariant derivative operator defined in the previous section and $U_{k}$ are a set of momentum-independent functions. The supertrace acting on $Q$, which includes also the trace in momentum space, is given by

$$
\mathrm{STr} Q\left(P_{\mu}, U_{k}\right)= \pm \int \frac{\mathrm{d}^{d} p}{(2 \pi)^{d}}\left\langle p\left|\operatorname{tr} Q\left(P_{\mu}, U_{k}\right)\right| p\right\rangle
$$

where $+(-)$ is for bosonic (fermionic) degrees of freedom, and tr denotes the trace only over internal degrees of freedom, e.g. gauge, spin, flavor, etc. It is convenient to use the

\footnotetext{
${ }^{4}$ In the fermionic case, we have $\Delta \sim p^{-1} \sim m_{H}^{-1}$, while $X$ can be at most of $\mathcal{O}(1)$. On the other hand, in the bosonic case, $\Delta \sim p^{-2} \sim m_{H}^{-2}$ while the interactions can be at most of $\mathcal{O}\left(m_{H}\right)$. Note that this counting holds even if $\mathcal{L}_{\mathrm{UV}}$ is itself an EFT, since the EFT validity requires $p, m_{H} \ll \Lambda$ for $\Lambda$ being the EFT cut off.

${ }^{5}$ Note that log-type traces with light-field propagators do not contain any heavy scales and, hence, only produce soft contributions.
} 
completeness relation of position states, $\int \mathrm{d}^{d} x|x\rangle\langle x|=\mathbb{1}$, to express $Q$ in position space:

$$
\begin{aligned}
& \mathrm{S} \operatorname{Tr} Q\left(P_{\mu}, U_{k}\right)= \pm \int \mathrm{d}^{d} x \int \frac{\mathrm{d}^{d} p}{(2 \pi)^{d}} e^{i p x} \operatorname{tr} Q\left(P_{\mu}, U_{k}(x)\right) e^{-i p x} \\
& = \pm \int \mathrm{d}^{d} x \int \frac{\mathrm{d}^{d} p}{(2 \pi)^{d}} \operatorname{tr} Q\left(P_{\mu}+p_{\mu}, U_{k}(x)\right) .
\end{aligned}
$$

In its current form, this expression is not manifestly covariant. At this point, it is useful to apply a path integral transformation, the so-called CDE expansion [31-33], that makes this expression manifestly covariant by putting all instances of $P_{\mu}$ into commutators of the form $\left[P_{\mu}, P_{\nu}\right],\left[P_{\mu},\left[P_{\nu}, P_{\rho}\right]\right],\left[P_{\mu}, U_{k}\right]$, etc. The CDE expansion consists in sandwiching the expression in (2.13) between the operators $e^{-P \cdot \partial_{p}}$ and $e^{P \cdot \partial_{p}}$ :

$$
\mathrm{S} \operatorname{Tr} Q\left(P_{\mu}, U_{k}\right)= \pm \int \mathrm{d}^{d} x \int \frac{\mathrm{d}^{d} p}{(2 \pi)^{d}} e^{-P \cdot \partial_{p}} \operatorname{tr} Q\left(P_{\mu}+p_{\mu}, U_{k}(x)\right) e^{P \cdot \partial_{p}}
$$

where $\partial_{p}$ denotes the partial derivative with respect to the loop momentum $p$. The operator $e^{P \cdot \partial_{p}}$ is trivially unity when acting to the right, while the operator $e^{-P \cdot \partial_{p}}$ also becomes unity when it is made to act from the left due to the vanishing of total derivatives under integration, so the supertrace remains invariant under this operation. ${ }^{6}$ However, when passing $e^{-P \cdot \partial_{p}}$ through $Q$ to cancel against $e^{P \cdot \partial_{p}}$, it has the desired effect of putting all $P$ 's into commutators. More precisely, this transformation yields

$$
\begin{aligned}
e^{-P \cdot \partial_{p}}\left(p_{\mu}+P_{\mu}\right) e^{P \cdot \partial_{p}} & =p_{\mu}+i \tilde{G}_{\mu \nu} \partial_{p}^{\nu} \\
\tilde{U}_{k} \equiv e^{-P \cdot \partial_{p}} U_{k} e^{P \cdot \partial_{p}} & =\sum_{n=0}^{\infty} \frac{(-i)^{n}}{n !}\left(D_{\left\{\alpha_{1}, \ldots \alpha_{n}\right\}} U_{k}\right) \partial_{p}^{\alpha_{1}} \cdots \partial_{p}^{\alpha_{n}},
\end{aligned}
$$

where the parenthesis denotes that the derivatives act in commutators as per usual, e.g. $\left(D_{\mu} A\right) \equiv\left[D_{\mu}, A\right],\left(D_{\mu} D_{\nu} A\right) \equiv\left[D_{\mu},\left[D_{\nu}, A\right]\right]$, etc., and

$$
\tilde{G}_{\mu \nu} \equiv \sum_{n=0}^{\infty} \frac{(-i)^{n}}{(n+2) n !}\left(D_{\left\{\alpha_{1}, \ldots \alpha_{n}\right\}} G_{\mu \nu}\right) \partial_{p}^{\alpha_{1}} \cdots \partial_{p}^{\alpha_{n}}, \quad D_{\left\{\mu_{1}, \cdots \mu_{n}\right\}} \equiv \frac{1}{n !} \sum_{\sigma \in \mathcal{S}_{n}} D_{\mu_{\sigma(1)}} \cdots D_{\mu_{\sigma(n)}} .
$$

Since $Q\left(P_{\mu}, U_{k}(\hat{x})\right)$ has a well-defined rational expansion in its arguments this implies

$$
\operatorname{STr} Q\left(P_{\mu}, U_{k}\right)= \pm \int \mathrm{d}^{d} x \int \frac{\mathrm{d}^{d} p}{(2 \pi)^{d}} \operatorname{tr} Q\left(p_{\mu}+i \tilde{G}_{\mu \nu} \partial_{p}^{\nu}, \tilde{U}_{k}(x)\right)
$$

yielding the desired manifestly covariant expression for the supertrace of $Q$.

\subsection{Explicit evaluation of the relevant supertraces}

In this section, we outline how to apply the covariant method to the log- and power-type supertraces.

\footnotetext{
${ }^{6}$ This invariance does not rely on the cyclic property of the trace, which has already been evaluated for momentum coordinates.
} 


\subsubsection{Log-type supertraces}

For the log-type supertraces, we have to evaluate $\left.\mathrm{S} \operatorname{Tr} \ln \Delta_{\eta_{H}}^{-1}\right|_{\text {hard }}$ for all possible $\eta_{H}$ propagators defined in (2.4). To apply the covariant supertrace evaluation in (2.17), we need to show first that $Q\left(P_{\mu}\right)=\ln \Delta_{\eta_{H}}^{-1}\left(P_{\mu}\right)$ satisfies the requirement of having a well-defined expansion in $P_{\mu}$. This can be shown by writing an integral representation of the logarithm:

$$
\ln \Delta_{\eta_{H}}^{-1}=\int_{z}^{1} \mathrm{~d} \xi \frac{\mathrm{d}\left(\Delta_{\eta_{H}}^{\xi}\right)^{-1}}{\mathrm{~d} \xi} \Delta_{\eta_{H}}^{\xi}-\ln \Delta_{\eta_{H}}^{z}
$$

with $\Delta_{i}^{\xi}$ defined as $\Delta_{i}$ in (2.4) but replacing $M_{i}$ by $\xi M_{i}$, such that $\mathrm{d}\left(\Delta_{\eta_{H}}^{\xi}\right)^{-1} / \mathrm{d} \xi$ does not depend on $p$. By taking the $z \rightarrow \infty$ limit, it is clear that $Q=\ln \Delta_{\eta_{H}}^{-1}$ can be expanded in inverse powers of $P_{\mu}$ and $M_{\eta_{H}}$ up to an infinite constant, $\ln \left(\Delta_{\eta_{H}}^{\infty}\right)$, that will be removed later. Hence, we can apply the covariant expression of the supertrace in (2.17) giving

$$
\mathrm{S} \operatorname{Tr} \ln \Delta_{\eta_{H}}^{-1}= \pm \int \mathrm{d}^{d} x \int \frac{\mathrm{d}^{d} p}{(2 \pi)^{d}} \operatorname{tr}\left\{\int_{\infty}^{1} \mathrm{~d} \xi \frac{\mathrm{d}\left(\Delta_{\eta_{H}}^{\xi}\right)^{-1}}{\mathrm{~d} \xi} \Delta_{\eta_{H}}^{\xi}\left(p_{\mu}+i \tilde{G}_{\mu \nu}\right)-\ln \left(\Delta_{\eta_{H}}^{\infty}\right)\right\}
$$

Since we are after the hard part of this trace, we can Taylor expand $\Delta_{\eta_{H}}^{\xi}\left(p_{\mu}+i \tilde{G}_{\mu \nu}\right)$ to remove $\tilde{G}_{\mu \nu}$ from the argument. We have

$$
\left.\mathrm{S} \operatorname{Tr} \ln \Delta_{\eta_{H}}^{-1}\right|_{\text {hard }}= \pm \int \mathrm{d}^{d} x \int \frac{\mathrm{d}^{d} p}{(2 \pi)^{d}} \operatorname{tr}\left\{\int_{\infty}^{1} \mathrm{~d} \xi \frac{\mathrm{d}\left(\Delta_{\eta_{H}}^{\xi}\right)^{-1}}{\mathrm{~d} \xi} \Delta_{\eta_{H}}^{\xi} \sum_{n=1}^{\infty}\left(\mathcal{G}_{\eta_{H}} \Delta_{\eta_{H}}^{\xi}\right)^{n}\right\}
$$

where we subtracted the $n=0$ term of the series and the infinite constant, which combine to give $\ln \Delta_{\eta_{H}}^{-1}$ and cancel against the path integral normalization factor. In this expression, we omitted the argument of $\Delta_{\eta_{H}}^{\xi}\left(p_{\mu}\right)$ for notational simplicity and defined

$$
\mathcal{G}_{i}= \begin{cases}-i\left\{p^{\mu}, \tilde{G}_{\mu \nu}\right\} \partial_{p}^{\nu}+\left(\tilde{G}_{\mu \nu} \partial_{p}^{\nu}\right)^{2} & \text { (scalar) } \\ -i \gamma^{\mu} \tilde{G}_{\mu \nu} \partial_{p}^{\nu} & \text { (fermion) } \\ +i\left\{p^{\mu}, \tilde{G}_{\mu \nu}\right\} \partial_{p}^{\nu}-\left(\tilde{G}_{\mu \nu} \partial_{p}^{\nu}\right)^{2} & \text { (vector) }\end{cases}
$$

The remaining evaluation of this supertrace is rather arduous but nevertheless straightforward, since the integral in $\xi$ is trivial after performing the well-known loop integrals

$$
\begin{aligned}
\mu^{2 \epsilon} & \int \frac{\mathrm{d}^{d} p}{(2 \pi)^{d}} \frac{p_{\mu_{1}} \cdots p_{\mu_{2 k}}}{\left(p^{2}-M^{2}\right)^{\alpha} p^{2 \beta}} \\
& =g_{\mu_{1} \ldots \mu_{2 k}} \frac{(-1)^{\alpha+\beta+k} i}{(4 \pi)^{2}} M^{2(2+k-\alpha-\beta)}\left(\frac{\bar{\mu}^{2} e^{\gamma_{E}}}{M^{2}}\right)^{\epsilon} \frac{\Gamma\left(\frac{d}{2}+k-\beta\right) \Gamma\left(\alpha+\beta-\frac{d}{2}-k\right)}{2^{k} \Gamma(\alpha) \Gamma\left(\frac{d}{2}+k\right)} .
\end{aligned}
$$


The evaluation of the log-type traces up to dimension six was done e.g. in [57]. For completeness, we list them here up to $\mathcal{O}\left(M_{i}^{-2}\right)$ :

$$
\begin{aligned}
\left.\frac{i}{2} \operatorname{STr} \ln \Delta_{\Phi, c_{V}}^{-1}\right|_{\text {hard }} & =\mp \frac{1}{16 \pi^{2}} \operatorname{tr}\left\{\frac{1}{12} \ln \frac{\bar{\mu}^{2}}{M_{\Phi, c_{V}}^{2}} G_{\mu \nu}^{2}+\frac{1}{M_{\Phi, c_{V}}^{2}}\left(\frac{1}{60}\left(D^{\mu} G_{\mu \nu}\right)^{2}+\frac{i}{90} G^{\mu}{ }_{\nu} G^{\nu}{ }_{\rho} G^{\rho}{ }_{\mu}\right)\right\}, \\
\left.\frac{i}{2} \mathrm{~S} \operatorname{Tr} \ln \Delta_{\Psi}^{-1}\right|_{\text {hard }} & =-\frac{1}{16 \pi^{2}} \operatorname{tr}\left\{\frac{1}{3} \ln \frac{\bar{\mu}^{2}}{M_{\Psi}^{2}} G_{\mu \nu}^{2}+\frac{1}{M_{\Psi}^{2}}\left(\frac{2}{15}\left(D^{\mu} G_{\mu \nu}\right)^{2}-\frac{i}{45} G^{\mu}{ }_{\nu} G_{\rho}^{\nu} G^{\rho}{ }_{\mu}\right)\right\}, \\
\left.\frac{i}{2} \mathrm{~S} \operatorname{Tr} \ln \Delta_{V}^{-1}\right|_{\text {hard }}= & \frac{1}{16 \pi^{2}} \operatorname{tr}\left\{\frac{1}{12}\left(1-2 \ln \frac{\bar{\mu}^{2}}{M_{V}^{2}}\right) G_{\mu \nu}^{2}\right. \\
- & \left.\frac{1}{M_{V}^{2}}\left(\frac{1}{30}\left(D^{\mu} G_{\mu \nu}\right)^{2}+\frac{i}{45} G^{\mu}{ }_{\nu} G^{\nu}{ }_{\rho} G^{\rho}{ }_{\mu}\right)\right\},
\end{aligned}
$$

where we removed the divergences in the dimension-four terms using the $\overline{\mathrm{MS}}$ scheme. They can be trivially recovered by taking $\ln \mu^{2} / M_{\eta_{H}}^{2} \rightarrow \ln \mu^{2} / M_{\eta_{H}}^{2}+1 / \epsilon($ for $d=4-2 \epsilon$ ).

\subsubsection{Power-type supertraces}

For the power-type traces, it is simpler to show that $Q\left(P_{\mu}, U_{k}\right)=(\Delta X)^{n}$ are indeed expansions of rational functions in $P_{\mu}$ and momentum-independent terms. Indeed, this is clearly the case for $\Delta\left(P_{\mu}\right)$, while in local theories the $X$ interactions can be written as

$$
X\left(P_{\mu}, \hat{x}\right)=\sum_{n=0}^{\infty} X_{n}^{\mu_{1} \cdots \mu_{n}}(\hat{x}) P_{\mu_{1}} \cdots P_{\mu_{n}},
$$

where $X_{n}$ are functions of fields and derivatives of fields acting inside commutators, such as $\left[P_{\mu}, \phi\right]=i\left(D_{\mu} \phi\right)$. The $P_{\mu}$ terms in the $X$ expansion are usually termed as "open covariant derivatives". Note that the expansion of $X$ as a polynomial in $P_{\mu}$ is not unique, since terms of the form $\left[P_{\mu}, \phi\right]$ always can be arranged as $\left[P_{\mu}, \phi\right]=P_{\mu} \phi-\phi P_{\mu}$. We fix this ambiguity by arranging the $P_{\mu}$ operators always to the rightmost.

Having argued that $Q\left(P_{\mu}, U_{k}\right)=(\Delta X)^{n}$ are expansions of rational functions in $P_{\mu}$ and $X_{n}^{\mu_{1} \cdots \mu_{n}}(\hat{x})$, we can apply the covariant expression of the supertrace in (2.17) giving

$$
\operatorname{STr}\left[(\Delta X)^{n}\right]= \pm \int \mathrm{d}^{d} x \int \frac{\mathrm{d}^{d} p}{(2 \pi)^{d}} \operatorname{tr}\left\{\left[\Delta\left(p_{\mu}+i \tilde{G}_{\mu \nu} \partial_{p}^{\nu}\right) \tilde{X}\right]^{n}\right\}
$$

with $\tilde{X} \equiv X\left(p_{\mu}+i \tilde{G}_{\mu \nu} \partial_{p}^{\nu}, \tilde{X}_{n}^{\mu_{1} \cdots \mu_{n}}(x)\right)$. Once more, we can benefit from only needing the hard part of the supertrace to expand out the $\tilde{G}_{\mu \nu}$ terms in the propagators, namely,

$$
\left.\mathrm{S} \operatorname{Tr}\left[(\Delta X)^{n}\right]\right|_{\text {hard }}= \pm \int \mathrm{d}^{d} x \int \frac{\mathrm{d}^{d} p}{(2 \pi)^{d}} \operatorname{tr}\left\{\left[\Delta \sum_{m=0}^{\infty}(\mathcal{G} \Delta)^{m} \tilde{X}\right]^{n}\right\},
$$

where $\Delta\left(p_{\mu}\right)$ are the free propagators defined in (2.4), and $\mathcal{G}$ is defined in (2.21). As with the log-type supertrace, the remaining evaluation of the power-type supertrace is straightforward and the loop integrals can be readily evaluated with

$$
\begin{aligned}
& \int \frac{\mathrm{d}^{d} p}{\left(2 \pi^{d}\right)} \frac{p_{\mu_{1}} \cdots p_{\mu_{2 k}}}{\left(p^{2}-M_{1}^{2}\right)^{\alpha_{1}} \cdots\left(p^{2}-M_{n}^{2}\right)^{\alpha_{n}} p^{2 \beta}} \\
& \quad=\sum_{m=1}^{n} \sum_{k=0}^{\alpha_{m}-1} \frac{1}{k !} \int \frac{\mathrm{d}^{d} p}{\left(2 \pi^{d}\right)} \frac{p_{\mu_{1}} \cdots p_{\mu_{2 k}}}{\left(p^{2}-M_{m}^{2}\right)^{\alpha_{m}-k} p^{2 \beta}}\left(\frac{\partial}{\partial M_{m}^{2}}\right)^{k} \prod_{\ell \neq m} \frac{1}{\left(M_{m}^{2}-M_{\ell}^{2}\right)^{\alpha_{\ell}}},
\end{aligned}
$$


along with formula (2.22). However, the amount of algebra involved in evaluating these supertraces makes it rather tedious without the use of computer tools.

\subsection{Comments on the treatment of $\gamma_{5}$ in fermion supertraces}

There is an added complication in the evaluation of fermion supertraces in terms where all propagators are fermionic, resulting in traces of $\gamma$-matrices. One of the primary outstanding problems in dimensional regularization is how to continue the definition of $\gamma_{5}$ away from 4 dimensions, cf. [58]. Whatever regularization procedure (and renormalization scheme) is used in the matching calculation requires the same choice to be used in subsequent computations in the EFT. We therefore propose to use a semi-naive implementation of dimensional regularization, as Naive Dimensional Regularization (NDR) often is the most practical choice for perturbative calculations.

For the Dirac algebra we formally set

$$
\left\{\gamma^{\mu}, \gamma^{\nu}\right\}=2 g^{\mu \nu}, \quad\left\{\gamma^{\mu}, \gamma_{5}\right\}=0, \quad \text { and } \quad \gamma_{5}^{2}=\mathbb{1},
$$

where all Lorentz indices are $d$-dimensional. This algebra in conjunction with cyclicity of the trace results in the vanishing of all traces with an odd number of $\gamma_{5}$ 's, prohibiting the recovery of the four-dimensional result in the limit $d \rightarrow 4$. We therefore abandon the cyclicity of $\gamma_{5}$-odd traces, while formally substituting $[59,60]$

$$
\operatorname{tr}\left[\gamma^{\mu} \gamma^{\nu} \gamma^{\rho} \gamma^{\sigma} \gamma_{5}\right]=-4 i \varepsilon^{\mu \nu \rho \sigma}+\mathcal{O}(\epsilon)
$$

With this prescription the choice of where the $\gamma_{5}$-odd traces are read from - meaning which $\gamma$ in a Dirac trace is written as the left-most — results in another $\mathcal{O}(\epsilon)$ ambiguity. This ambiguity is therefore only manifest in divergent diagrams, where the $\epsilon$ pole and the $\mathcal{O}(\epsilon)$ trace ambiguity combines to give a finite ambiguity in the computation.

The calculation of the one-loop effective action in the UV theory does not involve any UV divergent $\gamma_{5}$-odd diagram due to anomaly cancellation and the prescription is unambiguous. A complication arises when performing the matching computation and identifying $\mathcal{L}_{\text {EFT }}^{(1)}$ with the hard part of the functional supertrace: the expansion of the loop integral in heavy masses and hard loop momenta can introduce spurious IR divergences in some of the integrals. The IR divergences combined with the reading-point ambiguity introduce an ambiguity in $\mathcal{L}_{\mathrm{EFT}}^{(1)}$. Conveniently, when expanding loop integrals by regions, spurious IR divergences in the hard part of the integral are known to cancel exactly against corresponding UV divergences in the soft part, which in our case corresponds to 1-loop diagrams in the EFT. With the $\epsilon$ poles canceling in $\Gamma_{\mathrm{EFT}}^{(1)}$, so too will the ambiguities in $\gamma_{5}$-odd diagrams, as long as the $\gamma_{5}$ prescription is applied consistently between the matching and the EFT calculations. That is, as long as the reading points are chosen identically.

The consistent choice of reading point is perhaps best illustrated with an example. If the UV theory involves both light and heavy fermions, $\psi$ and $\Psi$, the effective action can contain contributions of the form

$$
\Gamma_{\mathrm{UV}}^{(1)} \supset-\frac{i}{2} \mathrm{~S} \operatorname{Tr}\left[\Delta_{\psi} X_{\psi \Psi} \Delta_{\Psi} X_{\Psi \psi}\right]
$$


In the UV theory the $\gamma_{5}$-odd piece of this part of the effective action is finite, thereby ensuring that there is no ambiguity from the reading point of the Dirac trace, which is embedded in the supertrace. Computing equivalent one-loop amplitudes in the EFT will involve a part coming from one-loop contributions to $S_{\mathrm{EFT}}$ and one loop diagrams with the tree-level EFT:

$$
\Gamma_{\mathrm{EFT}}^{(1)} \supset S_{\mathrm{EFT}}^{(1)}-\frac{i}{2} \mathrm{~S} \operatorname{Tr}\left[\Delta_{\psi} X_{\psi \psi}^{\mathrm{EFT}}\right]
$$

where the corresponding contribution to the EFT fluctuation operator quickly is identified as

$$
X_{\psi \psi}^{\mathrm{EFT}} \supset X_{\psi \Psi} \frac{i \not D+M_{\Psi}}{M_{\Psi}^{2}} X_{\Psi \psi}+\ldots
$$

The two contributions to $\Gamma_{\mathrm{EFT}}^{(1)}$ are readily identified with the hard and soft part of the UV loops, respectively. Consequently, the $\epsilon$ poles cancel between them and, when the same reading point is chosen, so will the reading point ambiguity in the finite part of the effective action. The reading point can be fixed by e.g. making sure that $X_{\Psi \psi}$ is the last piece of the trace, in both EFT computation and matching computations.

\subsection{Summary of the functional matching method}

In this section, we summarize the relevant steps needed to perform functional EFT matching at the one-loop level. These are:

i) Collecting all fields, heavy and light, into field multiplets: to obtain the fluctuation operator (2.3), one needs to take functional derivatives with respect to all fields in the theory, including field conjugates in the case of complex fields. For this reason, it is useful to arrange the fields into field multiplets

$$
\varphi_{\phi}=\left(\begin{array}{c}
\phi \\
\phi^{*}
\end{array}\right), \quad \varphi_{\psi}=\left(\begin{array}{c}
\psi \\
\psi^{c}
\end{array}\right), \quad \varphi_{A}=\left(\begin{array}{c}
A_{\mu} \\
A_{\mu}^{*}
\end{array}\right),
$$

in the case of complex scalars, fermions, and complex vectors, respectively. Here $f^{c}=$ $C \bar{f}^{\top}$ is the charge-conjugated fermion with $C$ being the charge conjugation matrix and both $f$ and $f^{c}$ 4-component Dirac spinors. In the case when only some chiralities are present, like in the SM, chiral projectors should be used in the corresponding interactions. Furthermore, it is convenient to organize the fields (in the form of (2.33)) into one field multiplet for each field type. These types are heavy scalar, light scalar, heavy fermion, light fermion, heavy vector, light vector, heavy ghost, and light ghost, which we generically denote by $\Phi, \phi, \Psi, \psi, V, A, c_{V}$, and $c_{A}$, respectively.

ii) Obtaining the heavy field EOMs: the EOMs can be determined directly by setting the first functional derivative of the UV Lagrangian with respect to the heavy fields equal to zero, namely

$$
\left.\frac{\delta \mathcal{L}_{\mathrm{UV}}}{\delta \eta_{H}}\right|_{\eta=\hat{\eta}}=0
$$

where we remind the reader that the hat denotes field configurations that satisfy the EOMs, and $\eta_{H}$ contains all the heavy fields multiplets $\eta_{\Phi}, \eta_{\Psi}, \eta_{V}$, and/or $\eta_{c_{V}}$ 
of the theory. These equations need to be expanded to a given order in the heavy mass expansion, matching the desired order in the EFT expansion, to obtain orderby-order expressions of the heavy fields in terms of light fields. The tree-level EFT Lagrangian is obtained by replacing these expressions into the UV Lagrangian.

iii) Determining the $\boldsymbol{X}$ terms: these are obtained from the second functional derivative of the UV Lagrangian with respect to heavy and light fields after subtraction of the inverse propagators. More precisely,

$$
X_{i j}=\delta_{i j} \Delta_{i}^{-1}-\left.\frac{\delta^{2} \mathcal{L}_{\mathrm{UV}}}{\delta \bar{\eta}_{i} \delta \eta_{j}}\right|_{\eta=\hat{\eta}},
$$

with the inverse propagators given in (2.4). Light-particle masses are always included in the $X$ terms to better organize the power counting. If the $X$ terms contain derivative interactions, these should be arranged in the form of (2.24). In most practical cases, only the terms $X_{0}$ and/or $X_{1}^{\mu}$ of this expansion are present. It is convenient to keep track of the mass dimension of the fields and derivatives acting on fields (e.g. "close covariant derivatives") inside each $X$ term, since this provides a simple power counting for the EFT expansion.

iv) Identifying and evaluating the relevant supertraces: the next step is to identify the relevant log-type and power-type supertraces that enter into the one-loop EFT matching equation (2.11). Log-type supertraces are model-independent, since they do not depend on the $X$ terms (encoding the relevant $\mathcal{L}_{\mathrm{UV}}$ interactions), but only on the heavy-field propagators. They can be evaluated from the expansion (2.20). A list of all log-type supertraces evaluated up to operators of dimension six is given in (2.23). A log-type supertrace should be included for each of the heavy fields in the theory, including the complex conjugate in the case of complex fields.

Power-type supertraces do depend on the $X$ terms and should (a priori) be computed for every UV model. The infinite series in (2.11), and hence the number of supertraces to compute, is truncated by the desired mass dimension of the EFT operators, which is determined by adding the mass dimensions of each of the $X$ terms appearing in a given supertrace. An important subtlety to consider is that the series (2.11) gives rise to symmetry factors in some of the supertraces. These symmetry factor are given by the inverse of the number of times the trace repeats itself under cyclic permutations. These types of supertraces can be evaluated by means of the expansion in (2.26).

The procedure presented here closely follows the prescription presented in [53]. However, there are a number of differences between the two. First, light-field masses are included in the $X$ terms and not in the propagators. Second, the derivative expansion of $X$ is defined such that all derivatives are made to act to the rightmost, cf. (2.24). Furthermore, we do not adopt a diagrammatic description for the identification of the relevant power-type supertraces, since this task is performed automatically by SuperTracer, as we describe below. 


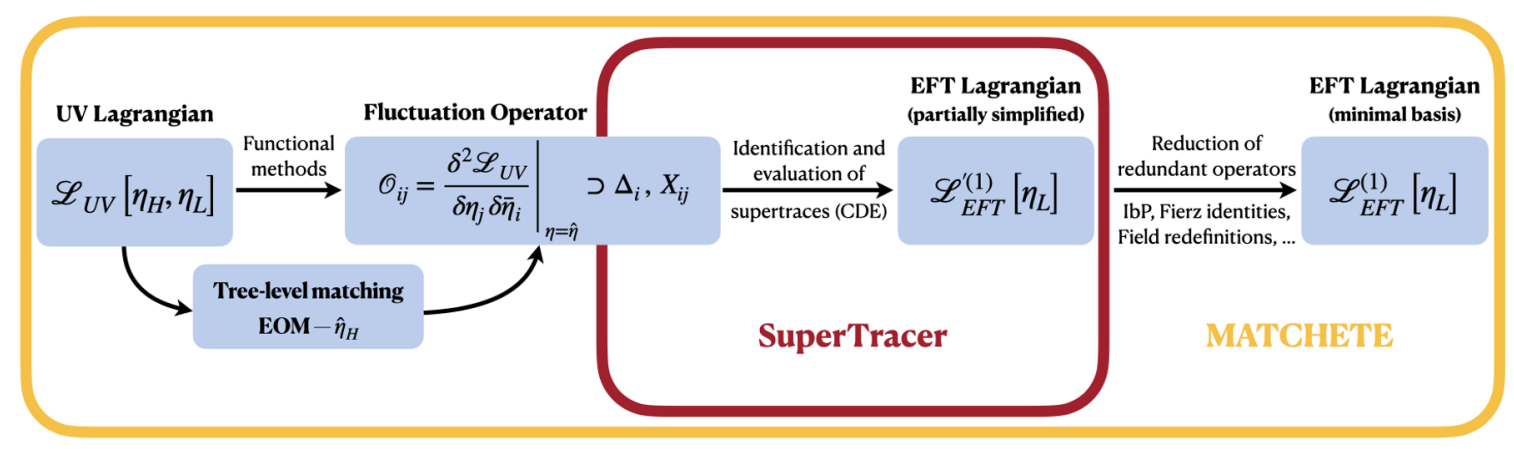

Figure 1. Summary of the functional approach to one-loop EFT matching. Highlighted in red, we show the parts of the procedure that are taken care of by SuperTracer, and in yellow those parts that will be handled by the master program MATCHETE (to be released).

\section{SuperTracer in a nutshell}

SuperTracer is a Mathematica package aimed at facilitating the functional EFT matching procedure described in section 2 and illustrated in figure 1 . The package takes over the most tedious parts of this procedure by automating the process of identifying and evaluating all relevant supertraces. The program also partially simplifies the resulting operators, as described below. However, it does not provide a complete reduction to a basis, and the calculation of the $X$ interactions and heavy field EOMs still has to be done manually. We delegate these tasks to the Mathematica package MATCHETE (Matching Effective Theories Efficiently) [61], which we are currently developing, and which will include SuperTracer at its core. The ultimate goal is to fully automate the matching procedure, having as input a user-defined UV Lagrangian, and completely eliminating the need for manually determining and inserting the $X$ interactions.

The main routines in the current implementation of SuperTracer evaluate log- and power-type supertraces by performing the following steps:

i) The propagators are reconstructed from the input list of $X$ interactions, and everything is placed in a non-commutative product. Fermionic traces are assigned an extra factor of $(-1)$.

ii) The covariant expansion of $\Delta$ and $X$ terms are performed to the appropriate order. All momentum derivatives act through the expression to terminate on the right.

iii) All Dirac products are simplified and matched to a basis of anti-symmetrized products, $\Gamma_{\mu_{1}, \ldots \mu_{n}}=\gamma_{\left[\mu_{1}\right.} \cdots \gamma_{\left.\mu_{n}\right]}$, and the loop integrals are evaluated using dimensional regularization with $d=4-2 \epsilon$ in the $\overline{\mathrm{MS}}$ scheme.

Added utility is provided by allowing the user to substitute model-specific expressions in the $X$ operators, making it possible to directly perform additional simplifications such as evaluating Dirac traces.

As for validation of the package, we have cross-checked a variety of supertraces against the STrEAM package as kindly provided by the authors [54]. Furthermore, the two example models discussed in section 4 have allowed us to check the package against a sample diagrammatic computation (cf. appendix B) and previous literature. 


\subsection{Downloading and installing the package}

The SuperTracer package is free software under the terms of the GNU General Public License v3.0 and is publicly available in the GitLab repository

$$
\text { https://gitlab.com/supertracer/supertracer }
$$

The package can be installed in one of two ways:

i) Automatic installation: the simplest way to download and install SuperTracer is to run the following command in a Mathematica notebook:

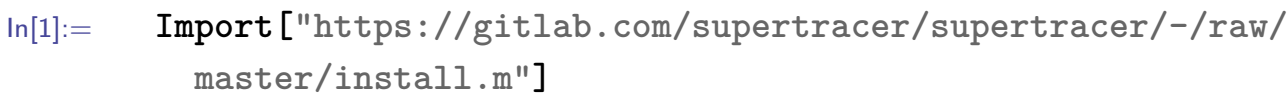

This will download and install SuperTracer in the Applications folder of Mathematica's base directory.

ii) Manual installation: the user can also manually download the package from the GitLab repository $\forall$. We recommend placing the SuperTracer folder in the Applications folder of Mathematica's base directory, so its location does not need be specified before loading the package. Alternatively, the user can place the SuperTracer folder in a different directory and specify its location via

$\ln [2]:=\quad$ AppendTo[\$Path, "directory"] ;

with directory being the path to the SuperTracer folder.

Once installed, the user can load SuperTracer in any Mathematica notebook by running $\ln [3]:=<$ SuperTracer

\section{StFFE

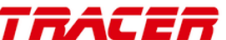

by Javier Fuentes-Martín, Matthias König, Julie Pagès, Anders Eller Thomsen, and Felix Wilsch Reference: arXiv:2012.08506

Website: https://gitlab.com/supertracer/supertracer

\subsection{SuperTracer global variables and routines}

After the package has been loaded, a variety of global variables and routines are defined. The main global variables are described in tables 1 and 2 . The routines available to the user are:

\section{Main SuperTracer routines}

- LogTerm[field, <order>] returns the log-type terms, resulting from integrating out a heavy field, to a given order in the EFT expansion. The field, which can be $\Phi, \Psi, \mathrm{V}$ or $\mathrm{cV}$, is assumed to be real (or Majorana in the fermionic case), so the output should be multiplied by two in the case of complex (or Dirac) fields. The order is assumed to be inclusive unless it is given inside curly 


\begin{tabular}{|c|c|}
\hline Variable & Description \\
\hline$\Phi, \phi, \Psi, \psi, \mathrm{V}, \mathrm{A}, \mathrm{cV}, \mathrm{cA}$ & $\begin{array}{l}\text { Field types. They are, respectively, heavy scalar, light scalar, } \\
\text { heavy fermion, light fermion, heavy vector, light vector, heavy } \\
\text { ghost, and light ghost. }\end{array}$ \\
\hline$X[\{f 1, f 2\},<o r d>]$ & $\begin{array}{l}\text { Input form for the } X \text { interactions. The arguments } f 1 \text { and } \\
f 2 \text { should be field types, while <ord> is an optional argument } \\
\text { specifying the order of } X \text {, which can be a single number (if } X_{0} \\
\text { is the only term in the expansion in }(2.24) \text { ) or a list of numbers } \\
\text { indicating the orders of the } X_{0,1,2, \ldots} \text { terms. If no <ord> is } \\
\text { given, the default values in Xords are taken. This variable } \\
\text { has a special output format, e.g. } \mathrm{X}[\{\psi, \psi\}] \text { shows as } \mathrm{X}_{\psi \psi} \text { and } \\
\mathrm{X}[\{\psi, \psi\}, 2] \text { as } \mathrm{X}_{\psi \psi}^{[2]} \text {. }\end{array}$ \\
\hline Xords & $\begin{array}{l}\text { Association with the default interaction order of each } X \text { term, } \\
\text { e.g. Xords } ₫\{\psi, \psi\} \text { returns } 1 \text {. }\end{array}$ \\
\hline $\mathrm{STr}[\mathrm{Xterms}]$ & $\begin{array}{l}\text { A header denoting a supertrace of the list Xterms of } X \text { interac- } \\
\text { tions. Symmetry factors and a global factor of }-\frac{i}{2} \text { is included } \\
\text { into the definition of this variable for notational simplicity. }\end{array}$ \\
\hline $\mathrm{M}[\mathrm{label}]$ & Heavy field mass. The output has special format $M_{\text {label }}$ \\
\hline \$DegenerateMasses & $\begin{array}{l}\text { Global variable that can be True or False (set to True by } \\
\text { default). If True, all heavy scales are assumed to be equal to } \\
\text { M["H"] (output format } \mathrm{M}_{\mathrm{H}} \text { ). }\end{array}$ \\
\hline$\mu$ bar2 & $\begin{array}{l}\text { Matching scale squared. This variable has the special output } \\
\text { format } \bar{\mu}^{2} \text {. }\end{array}$ \\
\hline
\end{tabular}

Table 1. Main SuperTracer variables.

brackets, e.g. LogTerm $[\Phi, 6]$ provides all operators up to dimension six, while $\operatorname{LogTerm}[\Phi,\{6\}]$ provides only operators of dimension six. The order is an optional argument. If no order is given, 6 is assumed.

- PowerTerms [Xterms, <order>] returns the sum of all power-type traces that need to be computed for a given list of $X$ terms to a given order in the EFT expansion. As for LogTerm, the order is an optional argument and it is treated in the same manner. For example, if we have a theory with three $X$ terms, $X_{\psi \Phi}^{[3 / 2]}, X_{\Phi \psi}^{[3 / 2]}$ and $X_{\psi \psi}^{[3]}$, by running PowerTerms

$$
\begin{array}{ll}
\operatorname{In}[4]:= & \text { PowerTerms }[\{\mathrm{X}[\{\psi, \Phi\}, 3 / 2], \mathrm{X}[\{\psi, \psi\}, 3]\}] \\
\text { Out }[4]= & \operatorname{STr}\left[\left\{\mathrm{X}_{\Phi \psi}^{[3 / 2]}, \mathrm{X}_{\psi \Phi}^{[3 / 2]}\right\}\right]+\operatorname{STr}\left[\left\{\mathrm{X}_{\Phi \psi}^{[3 / 2]}, \mathrm{X}_{\psi \psi}^{[3]}, \mathrm{X}_{\Psi \Phi}^{[3 / 2]}\right\}\right]+ \\
& \operatorname{STr}\left[\left\{\mathrm{X}_{\Phi \psi}^{[3 / 2]}, \mathrm{X}_{\psi \Phi}^{[3 / 2]}, \mathrm{X}_{\Phi \psi}^{[3 / 2]}, \mathrm{X}_{\psi \Phi}^{[3 / 2]}\right\}\right]
\end{array}
$$

we find that three supertraces, denoted by STr, need to be computed at the level of dimension six operators. Note that the symmetry factor $1 / 2$ that would appear in $\operatorname{STr}\left[\left\{\mathrm{X}_{\Phi \psi}^{[3 / 2]}, \mathrm{X}_{\psi \Phi}^{[3 / 2]}, \mathrm{X}_{\Phi \psi}^{[3 / 2]}, \mathrm{X}_{\psi \Phi}^{[3 / 2]}\right\}\right]$ (cf. section 2.4) and a global $-\frac{i}{2}$ is absorbed into the definition of $\mathrm{STr}$ for notational simplicity. Further note 
that conjugate interactions need not be introduced since these are automatically included by PowerTerms. Indeed, in our example we have input $\mathrm{X}[\{\psi, \Phi\}, 3 / 2]$ but not $\mathrm{X}[\{\Phi, \psi\}, 3 / 2]$.

- STrTerm [Xterms, <order $>,\langle$ Xsubstitutions $>$ evaluates the power-type supertrace of a given list of $X$ terms to a given order in the EFT expansion. The output of STrTerm is assumed to be inside $\int \mathrm{d}^{d} x \frac{1}{16 \pi^{2}} \operatorname{tr}[$.$] , with \operatorname{tr}$ [.] being a trace over internal degrees of freedom. Moreover, note that the definition of supertrace in SuperTracer includes symmetry factors and a global $-\frac{i}{2}$ factor. This routine further allows for the optional substitution of the $X$ terms into their explicit expressions in terms of fields. Rather than explaining how to perform $X$ substitutions here, we provide detailed usage examples of this functionality in section 4 and in the ancillary Mathematica notebooks. The order is treated in the same way as in the LogTerm routine. However, if the list of $X$ substitutions is given, the order must also be given. Finally, the $\epsilon$ poles are removed from the output of STrTerm. They can be easily recovered since their coefficient matches that of the renormalization-scale logarithm.

- SuperSimplify[expr] is the primary simplification routine, which provides a one-point-stop for simplifications of SuperTracer outputs. It simplifies outputs of STrTerm and LogTerm by attempting different index labels and collecting terms with the same operator structure. SuperSimplify also calls SimplifyOutput, which uses integration by parts, commutator, and Jacobi identities to match the output to a basis of operators. Although the outputs of SuperTracer is shown as a normal sum of terms to the user, its full Mathematica form consists of a sum of LTerm [coeff, OpStr] that separate the coefficient and operator structure of each term for better internal manipulations.

\section{Routines for $X$ substitutions}

- AddField[label, type, <charge ( $s$ )>,<countingDim>] defines a field of a given type (cf. table 1) with a given label, so it can be used in an $X$ substitution. If the field is charged under a single gauge $\mathrm{U}(1)$, its charge should be provided as label [charge], where label is a label for the U(1) symmetry chosen by the user and charge is a number specifying the field charge. On the other hand, if the field is charged under multiple $\mathrm{U}(1)$ gauge groups, the user should give a list of charges with the format \{label1 [charge1], labe12 [charge2], ... \}. As an example, let us define a heavy scalar field $f$ with charge 2 under a gauge $\mathrm{U}(1)_{L}$ that we label by $L$ :

$$
\ln [5]:=\quad \text { AddField }[f, \Phi, L[2]]
$$

This creates the field routine $f$ [Indices] where the flavor and gauge indices carried by the field should be given as a list. If the field carries no indices, no argument or an empty list can be given. In the case of a vector field, the first entry in the list must be a Lorentz index $\mu$, i.e. the indices should then be given in the format $\{\mu, \mathrm{rep} 1[$ ind1] ,rep2 [ind2] , . . $\}$, where rep1 denotes the 


\begin{tabular}{|c|c|}
\hline Variable & Description \\
\hline $\mathrm{g}[\mu, \nu]$ & $\begin{array}{l}\text { Lorentz metric tensor. The arguments } \mu, \nu \text { are } \\
\text { Lorentz indices. This variable has the special out- } \\
\text { put format } \mathrm{g}_{\mu \nu} \text {. }\end{array}$ \\
\hline$\varepsilon[\mu, \nu, \rho, \sigma]$ & $\begin{array}{l}\text { Levi-Civita symbol. The arguments } \mu, \nu, \rho, \sigma \text { are } \\
\text { Lorentz indices. This variable has the special out- } \\
\text { put format } \varepsilon_{\mu \nu \rho \sigma} \text {. }\end{array}$ \\
\hline$\gamma[\mu], \gamma[5]$ & $\begin{array}{l}\text { Dirac matrices. The argument } \mu \text { is a Lorentz index. } \\
\text { The output has the special form } \gamma_{\mu} \text { or } \gamma_{5} \text {. }\end{array}$ \\
\hline$P L, P R$ & $\begin{array}{l}\text { Chiral projectors. The output has the special output } \\
\text { form } P_{L} \text { and } P_{R} \text {, respectively. }\end{array}$ \\
\hline $\mathrm{T}[\{\operatorname{repA}[\mathrm{A}], \operatorname{rep}[\mathrm{a}], \operatorname{rep}[\mathrm{b}]\}]$ & $\begin{array}{l}\text { Symmetry generator. The arguments a and } \mathrm{b} \text { are } \\
\text { indices in the representation rep, whereas } \mathrm{A} \text { is in } \\
\text { the representation repA. The output has the spe- } \\
\text { cial form } \mathrm{T}^{\text {Aab }} \text {. }\end{array}$ \\
\hline $\mathrm{GA}[\{\mu, \nu\}, \operatorname{tag}]$ & $\begin{array}{l}\text { Field-strength tensor for Abelian groups. The ar- } \\
\text { guments } \mu \text { and } \nu \text { are Lorentz indices and tag is the } \\
\text { symbol used to label the Abelian symmetry. Abelian } \\
\text { field-strength tensors are displayed as } \mathrm{F}_{\text {tag }}^{\mu \nu} \text { in the out- } \\
\text { put. }\end{array}$ \\
\hline $\operatorname{GnA}[\{\mu, \nu\},\{\operatorname{rep}[a], \operatorname{rep}[b]\}]$ & $\begin{array}{l}\text { Field-strength tensor for non-Abelian groups. As } \\
\text { before, the arguments } \mu \text { and } \nu \text { are Lorentz indices, } \\
\text { while a and } \mathrm{b} \text { label non-Abelian group indices in rep- } \\
\text { resentation rep. Non-Abelian field-strength tensors } \\
\text { have output format } \mathrm{G}_{\mathrm{rep}}^{\text {ab } \mu \nu} \text {. }\end{array}$ \\
\hline$\delta[\{\operatorname{rep}[\mathrm{a}], \operatorname{rep}[\mathrm{b}]\}]$ & $\begin{array}{l}\text { Kronecker delta. The two arguments a and } \mathrm{b} \text { are } \\
\text { indices of the representation rep. This variable has } \\
\text { the special output format } \delta^{\mathrm{ab}} \text {. }\end{array}$ \\
\hline $\operatorname{eps}[\{\operatorname{rep}[a], \operatorname{rep}[b]\}]$ & $\begin{array}{l}\text { Anti-symmetric tensor with two indices. The two } \\
\text { arguments a and } \mathrm{b} \text { are indices of the representation } \\
\text { rep. This variable has the special output format } \epsilon_{\mathrm{rep}}^{\mathrm{ab}} \text {. }\end{array}$ \\
\hline Flavor [index] & $\begin{array}{l}\text { Flavor index. The header Flavor labels the flavor } \\
\text { representation and specifies that index is a flavor } \\
\text { index. }\end{array}$ \\
\hline
\end{tabular}

Table 2. SuperTracer variables relevant for $X$ substitutions.

representation of the index ind1. To remove the field $f$ from the set of defined fields, the routine RemoveField[f] can be used.

- Bar [obj] returns the bar of a fermion field or the complex conjugate of other fields. Applying Bar to couplings and generators yields their conjugate. The 
routine can also be applied to representations and charges in $X$ substitutions to denote their conjugate. This routine has a special output format, i.e. Bar [obj] shows as $\overline{\mathrm{obj}}$.

- Transp [obj] returns the transpose of any object in Dirac space, that is, fermion fields, chiral projectors, or Dirac matrices. This routine has a special output format, i.e. Transp [obj] shows as obj ${ }^{\mathrm{T}}$.

- CConj[field] returns the charge conjugate of a fermion field, e.g. CConj [f []] gives $\mathrm{CC} * * \overline{\mathrm{f}}^{\mathrm{T}}$ with $\mathrm{CC}$ being the charge conjugation matrix. If this routine is applied to something other than a fermion field, the output is aborted and a warning is issued.

- $\mathrm{CD}$ [index, expr] or $\mathrm{CD}[\{$ indices\}, expr] returns the covariant derivative(s) of a given expression, with the number of Lorentz indices determining the number of derivatives. If the covariant derivative acts on a undefined variable, it is assumed to be vanishing.

To keep track of non-commutative objects SuperTracer co-opts Mathematica's build in NonCommutativeMultiply $(* *)$. Field objects, elements of the Dirac algebra and fieldstrength tensors are treated as non-commutative until the end of the computation. Only when using the substitution capability of STrTerm is non-commutativity for bosonic fields and field-strength tensors relaxed. All substitution rules must be given as non-commutative products.

As we have already described in certain routines and global variables, we have defined special output formats for some expressions to facilitate the reading of SuperTracer outputs. The explicit Mathematica expression of the output can be seen by applying the InputForm/FullForm routine. Although understanding this explicit form is not necessary to use all SuperTracer features, it is required when doing further manipulations of the output. We refer the interested reader to appendix A for more details on the variables that are used there.

\section{Usage examples}

Here we illustrate the matching procedure described in section 2 and the functionality of the SuperTracer package with two examples of heavy field integration: a toy model with a heavy vector-like fermion and an $S_{1}$ scalar leptoquark extension of the SM.

\subsection{Toy model with a heavy vector-like fermion}

As a first example, we consider a toy model with a heavy fermion $\Psi$ charged under a gauged $\mathrm{U}(1)_{e}$ with a Yukawa interaction to a singlet scalar $\phi$ and the left-handed component of a light fermion $\psi$. The Lagrangian of the model is given by

$$
\mathcal{L}=-\frac{1}{4} F_{\mu \nu} F^{\mu \nu}+\frac{1}{2}\left(\partial^{\mu} \phi\right)\left(\partial_{\mu} \phi\right)+\bar{\psi} i \not D \psi+\bar{\Psi}(i \not D-M) \Psi-\left(y \bar{\psi}_{L} \phi \Psi_{R}+\text { h.c. }\right)+\mathcal{L}_{\xi},
$$


where $D_{\mu} \psi=\partial_{\mu} \psi-i e A_{\mu} \psi$ (similarly for $\Psi$ ) and $\mathcal{L}_{\xi}=-\left(\partial_{\mu} A^{\mu}\right)^{2} /(2 \xi)$ is the gaugefixing Lagrangian. We illustrate the functional integration of $\Psi$ up to one-loop order and dimension-six operators. The tree-level EFT Lagrangian is easily obtained by substituting the classical value of $\Psi$, defined by its EOM, into the model Lagrangian. The EOM for $\Psi$ reads

$$
\Psi=-\frac{1}{M} y^{*} \phi \psi_{L}-\frac{1}{M^{2}} y^{*} i \not D\left(\phi \psi_{L}\right)+\mathcal{O}\left(M^{-3}\right),
$$

where we ignored terms of $\mathcal{O}\left(M^{-3}\right)$, since they do not contribute to the matching of dimension-six operators, neither at tree-level nor at the one-loop order. After substituting (4.2) into the model Lagrangian, the tree-level EFT Lagrangian is given by

$$
\mathcal{L}_{\mathrm{EFT}}^{(0)}=-\frac{1}{4} F_{\mu \nu} F^{\mu \nu}+\frac{1}{2}\left(\partial^{\mu} \phi\right)\left(\partial_{\mu} \phi\right)+\bar{\psi} i \not D \psi+\mathcal{L}_{\xi}+\frac{|y|^{2}}{M^{2}}\left(\bar{\psi}_{L} \phi\right) i \not D\left(\phi \psi_{L}\right)+\mathcal{O}\left(M^{-4}\right) .
$$

Let us now proceed to the one-loop matching computation. As discussed in section 2, we fix $\xi=1$ for the quantum fluctuation. Next, we rewrite the fields into multiplets in the form of (2.33):

$$
\varphi_{\phi}=\phi, \quad \varphi_{A}=A_{\mu}, \quad \varphi_{\psi}=\left(\begin{array}{c}
\psi \\
\psi^{c}
\end{array}\right), \quad \varphi_{\Psi}=\left(\begin{array}{c}
\Psi \\
\Psi^{c}
\end{array}\right),
$$

with the $c$ superscript denoting charge conjugation. The $X$ terms for this Lagrangian read (cf. $(2.35)$ )

$$
\begin{array}{rlrl}
X_{\Psi A}^{[5 / 2]}=\left(\begin{array}{c}
-e \gamma_{\mu} \Psi \\
e \gamma_{\mu} \Psi^{c}
\end{array}\right), & X_{\psi A}^{[3 / 2]}=\left(\begin{array}{c}
-e \gamma_{\mu} \psi \\
e \gamma_{\mu} \psi^{c}
\end{array}\right), \quad X_{\psi \Psi}^{[1]}=\left(\begin{array}{cc}
y P_{R} & 0 \\
0 & y^{*} P_{L}
\end{array}\right) \phi, \\
X_{\psi \phi}^{[7 / 2]}=\left(\begin{array}{c}
y P_{R} \Psi \\
y^{*} P_{L} \Psi^{c}
\end{array}\right), & X_{\Psi \phi}^{[3 / 2]}=\left(\begin{array}{c}
y^{*} P_{L} \psi \\
y P_{R} \psi^{c}
\end{array}\right), &
\end{array}
$$

while the term with interchanged fields simply correspond to the conjugate of the ones displayed here, e.g. $X_{B A}=\bar{X}_{A B}$. As for the tree-level EFT Lagrangian, the heavy field $\Psi$ needs to be replaced by its classical configuration in (4.2), e.g.

$$
X_{\psi \phi}^{[7 / 2]}=-\frac{|y|^{2}}{M^{2}}\left(\begin{array}{c}
P_{R} i \not D(\phi \psi) \\
P_{L} i \not D\left(\phi \psi^{c}\right)
\end{array}\right)+\mathcal{O}\left(M^{-3}\right) .
$$

Note that we have added a superindex to the $X$ terms with the total mass dimension of the light fields and covariant derivatives in them. This provides a useful way for counting the mass dimension of a given supertrace. The full set of $X$ terms constitute the main input for SuperTracer to evaluate the one-loop effective Lagrangian.

As described in section 2, the one-loop functional determinant is divided into log-type and power-type contributions, namely $\mathcal{L}_{\mathrm{EFT}}^{(1)}=\mathcal{L}_{\log }^{(1)}+\mathcal{L}_{\text {power }}^{(1)}$. The log-type contribution is obtained from SuperTracer by calling the LogTerms routine:

$$
\begin{aligned}
& \operatorname{In}[6]:=\quad \log \operatorname{Term}[\Psi, 6] \\
& \text { Out }[6]=\quad-\frac{1}{6} \log \left[\frac{\bar{\mu}^{2}}{\mathrm{M}_{\mathrm{H}}^{2}}\right] \mathrm{G}^{\mu \nu} * * \mathrm{G}^{\mu \nu}+\frac{1}{15} \frac{1}{\mathrm{M}_{\mathrm{H}}^{2}} \mathrm{D}_{\mu} \mathrm{G}^{\mu \nu} * * \mathrm{D}_{\rho} \mathrm{G}^{\nu \rho}+\frac{1}{90} \mathrm{i} \frac{1}{\mathrm{M}_{\mathrm{H}}^{2}} \mathrm{G}^{\mu \nu} * * \mathrm{G}^{\mu \rho} * * \mathrm{G}^{\nu \rho}
\end{aligned}
$$


and multiplying the output by 2 as $\Psi$ is a Dirac fermion in this example. Since $\Psi$ is charged under an abelian symmetry, we have $\mathrm{G}_{\mu \nu}=e F_{\mu \nu}$, and the resulting Lagrangian reads

$$
\mathcal{L}_{\log }^{(1)}=\frac{e^{2}}{16 \pi^{2}}\left[-\frac{1}{3} \log \frac{\mu^{2}}{M_{\Psi}^{2}} F_{\mu \nu} F^{\mu \nu}-\frac{2}{15 M_{\Psi}^{2}} D_{\mu} F^{\mu \nu} D^{\rho} F_{\rho \nu}\right] .
$$

As described in section 2.2.2, for the power-type contributions, we have to evaluate all possible supertraces constructed out of powers of $\Delta_{i} X_{i j}$ blocks starting with a heavy field propagator, with the sum of $X$ term dimensions not exceeding the desired operator dimension of the EFT Lagrangian. For this example, the power-type Lagrangian up to dimension six is obtained from the following supertraces:

$$
\begin{aligned}
\int \mathrm{d}^{d} x \mathcal{L}_{\text {power }}^{(1)}= & -\frac{i}{2}\left[\operatorname{STr}\left\{\Delta_{\Psi} X_{\Psi A}^{[5 / 2]} \Delta_{A} X_{A \Psi}^{[5 / 2]}\right\}+\operatorname{STr}\left\{\Delta_{\Psi} X_{\Psi \phi}^{[3 / 2]} \Delta_{\phi} X_{\phi \Psi}^{[3 / 2]}\right\}\right. \\
& +\operatorname{S} \operatorname{Tr}\left\{\Delta_{\Psi} X_{\Psi \psi}^{[1]} \Delta_{\psi} X_{\psi \Psi}^{[1]}\right\}+\left(\operatorname{STr}\left\{\Delta_{\Psi} X_{\Psi A}^{[5 / 2]} \Delta_{A} X_{A \psi}^{[3 / 2]} \Delta_{\psi} X_{\psi \Psi}^{[1]}\right\}\right. \\
& \left.+\operatorname{STr}\left\{\Delta_{\Psi} X_{\Psi \psi}^{[1]} \Delta_{\psi} X_{\psi \phi}^{[7 / 2]} \Delta_{\phi} X_{\phi \Psi}^{[3 / 2]}\right\}+\text { h.c. }\right) \\
& +\operatorname{S} \operatorname{Tr}\left\{\Delta_{\Psi} X_{\Psi \psi}^{[1]} \Delta_{\psi} X_{\psi A}^{[3 / 2]} \Delta_{A} X_{A \psi}^{[3 / 2]} \Delta_{\psi} X_{\psi \Psi}^{[1]}\right\} \\
& +\operatorname{S} \operatorname{Tr}\left\{\Delta_{\Psi} X_{\Psi \psi}^{[1]} \Delta_{\psi} X_{\psi \Psi}^{[1]} \Delta_{\Psi} X_{\Psi \phi}^{[3 / 2]} \Delta_{\phi} X_{\phi \Psi}^{[3 / 2]}\right\} \\
& +\frac{1}{2} \operatorname{STr}\left\{\left(\Delta_{\Psi} X_{\Psi \phi}^{[3 / 2]} \Delta_{\phi} X_{\phi \Psi}^{[3 / 2]}\right)^{2}\right\}+\frac{1}{2} \operatorname{STr}\left\{\left(\Delta_{\Psi} X_{\Psi \psi}^{[1]} \Delta_{\psi} X_{\psi \Psi}^{[1]}\right)^{2}\right\} \\
& \left.+\frac{1}{3} \operatorname{STr}\left\{\left(\Delta_{\Psi} X_{\Psi \psi}^{[1]} \Delta_{\psi} X_{\psi \Psi}^{[1]}\right)^{3}\right\}\right]_{\text {hard }} \cdot
\end{aligned}
$$

The symmetry factors $1 / 2$ and $1 / 3$ appearing in front of some of the supertraces count the power of repeated blocks in a given supertrace. The same expression can be readily obtained from SuperTracer with the PowerTerms routine:

$$
\begin{aligned}
& \operatorname{In}[7]:=\operatorname{Xterms}=\{X[\{\Psi, A\}, 5 / 2], X[\{\psi, A\}, 3 / 2], X[\{\psi, \Psi\}, 1], X[\{\psi, \phi\}, 7 / 2], \\
& \mathrm{X}[\{\Psi, \phi\}, 3 / 2]\} \text {; } \\
& \text { LagPower }=\text { PowerTerms [Xterms, 6] } \\
& \text { Out }[7]=\operatorname{STr}\left[\left\{\mathrm{X}_{\Psi \mathrm{A}}^{[5 / 2]}, \mathrm{X}_{\mathrm{A \Psi}}^{[5 / 2]}\right\}\right]+\operatorname{STr}\left[\left\{\mathrm{X}_{\Psi \phi}^{[3 / 2]}, \mathrm{X}_{\phi \Psi}^{[3 / 2]}\right\}\right]+\operatorname{STr}\left[\left\{\mathrm{X}_{\Psi \psi}^{[1]}, \mathrm{X}_{\psi \Psi}^{[1]}\right\}\right]+ \\
& \operatorname{STr}\left[\left\{\mathrm{X}_{\Psi \mathrm{A}}^{[5 / 2]}, \mathrm{X}_{\mathrm{A} \psi}^{[3 / 2]}, \mathrm{X}_{\psi \Psi}^{[1]}\right\}\right]+\operatorname{STr}\left[\left\{\mathrm{X}_{\Psi \phi}^{[3 / 2]}, \mathrm{X}_{\phi \psi}^{[7 / 2]}, \mathrm{X}_{\psi \Psi}^{[1]}\right\}\right]+ \\
& \operatorname{STr}\left[\left\{\mathrm{X}_{\Psi \psi}^{[1]}, \mathrm{X}_{\psi \mathrm{A}}^{[3 / 2]}, \mathrm{X}_{\mathrm{A} \Psi}^{[5 / 2]}\right\}\right]+\operatorname{STr}\left[\left\{\mathrm{X}_{\Psi \psi}^{[1]}, \mathrm{X}_{\psi \phi}^{[7 / 2]}, \mathrm{X}_{\phi \Psi}^{[3 / 2]}\right\}\right]+ \\
& \operatorname{STr}\left[\left\{\mathrm{X}_{\Psi \phi}^{[3 / 2]}, \mathrm{X}_{\phi \Psi}^{[3 / 2]}, \mathrm{X}_{\Psi \phi}^{[3 / 2]}, \mathrm{X}_{\phi \Psi}^{[3 / 2]}\right\}\right]+\operatorname{STr}\left[\left\{\mathrm{X}_{\Psi \psi}^{[1]}, \mathrm{X}_{\psi \mathrm{A}}^{[3 / 2]}, \mathrm{X}_{\mathrm{A} \psi}^{[3 / 2]}, \mathrm{X}_{\psi \Psi}^{[1]}\right\}\right]+ \\
& \operatorname{STr}\left[\left\{\mathrm{X}_{\Psi \psi}^{[1]}, \mathrm{X}_{\psi \Psi}^{[1]}, \mathrm{X}_{\Psi \phi}^{[3 / 2]}, \mathrm{X}_{\phi \Psi}^{[3 / 2]}\right\}\right]+\mathrm{S} \operatorname{Tr}\left[\left\{\mathrm{X}_{\Psi \psi}^{[1]}, \mathrm{X}_{\psi \Psi}^{[1]}, \mathrm{X}_{\Psi \psi}^{[1]}, \mathrm{X}_{\psi \Psi}^{[1]}\right\}\right]+ \\
& \operatorname{STr}\left[\left\{\mathrm{X}_{\Psi \psi}^{[1]}, \mathrm{X}_{\psi \Psi}^{[1]}, \mathrm{X}_{\Psi \psi}^{[1]}, \mathrm{X}_{\psi \Psi}^{[1]}, \mathrm{X}_{\Psi \psi}^{[1]}, \mathrm{X}_{\psi \Psi}^{[1]}\right\}\right]
\end{aligned}
$$

This routine takes as input all $X$ terms present in a given model, defined in Xterms and the maximal mass dimension of the supertraces, which is 6 in our example. The routine PowerTerms automatically completes the list of $X$ interactions in Xterms with the corresponding conjugate interactions, namely $X_{A \psi}^{[5 / 2]}, X_{A \psi}^{[3 / 2]}$, etc. are automatically included, so their input is optional. Further note that the $-\frac{i}{2}$ and symmetry factors are absorbed in the 
definition of STr in SuperTracer. Moreover, the field propagators in between $X$ terms are implicitly understood. These supertraces can be evaluated by replacing STr by STrTerms. For concreteness, let us focus in the first term of this expression:

$$
\begin{aligned}
\operatorname{In}[8]:=\quad \begin{array}{l}
\text { LagPower }[[1]] \\
\% / \operatorname{STr} \rightarrow \operatorname{STrTerm}
\end{array} \\
\text { Out }[8]=\quad \operatorname{STr}\left[\left\{\mathrm{X}_{\Psi \mathrm{A}}^{[5 / 2]}, \mathrm{X}_{\mathrm{A} \Psi}^{[5 / 2]}\right\}\right] \\
\operatorname{Out}[9]=\frac{1}{8} \mathrm{i}\left(3+2 \log \left[\frac{\bar{\mu}^{2}}{\mathrm{M}_{\mathrm{H}}^{2}}\right]\right) \gamma_{\mu} * * \mathrm{D}_{\mu} \mathrm{X}_{\Psi_{\mathrm{i}} \mathrm{A}_{\mathrm{j}}} * * \mathrm{X}_{\mathrm{A}_{\mathrm{j}} \Psi_{\mathrm{i}}}+\frac{1}{2}\left(1+\log \left[\frac{\bar{\mu}^{2}}{\mathrm{M}_{\mathrm{H}}^{2}}\right]\right) \mathrm{M}_{\mathrm{H}} \mathrm{X}_{\Psi_{\mathrm{i}} \mathrm{A}_{\mathrm{j}}} * * \mathrm{X}_{\mathrm{A}_{\mathrm{j}} \Psi_{\mathrm{i}}}
\end{aligned}
$$

Note that, for notational simplicity, $\int \mathrm{d}^{d} x \frac{1}{16 \pi^{2}}$ is omitted in the output of STrTerms.

The SuperTracer package also allows for the substitution of the $X$ interactions. Let us continue to use the above term as an example. First, we need to define the fields appearing in a given $X$ term. Since in this case we want to replace $X_{\Psi A}$ in (4.5), we only need to define the heavy fermion field $\Psi$. This is done by calling the routine AddField:

$$
\ln [10]:=\operatorname{AddField}[\psi \mathrm{h}, \Psi, \quad \text { e[1] }]
$$

where the first argument is the label we are going to use for the field, the second argument is the type of field, and the third argument the $\mathrm{U}(1)_{e}$ charge. In this example, we are denoting the $\Psi$ field with the label ' $\psi_{h}$,' and we are defining it as a heavy fermion field, $\Psi$ in SuperTracer notation. Note that we have avoided using the label ' $\Psi$ ', since this variable is already predefined in SuperTracer. Once the field has been defined, we can introduce the value of $X_{\Psi A}$ in (4.5) (and its conjugate) into the STrTerm routine:

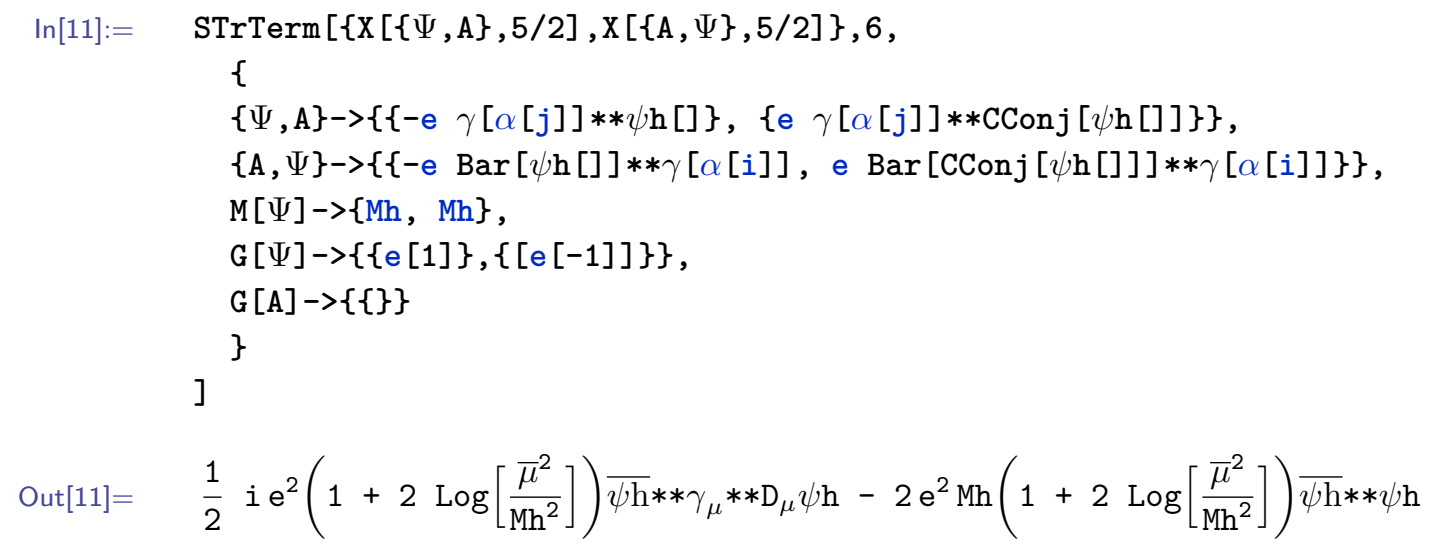

A few comments on the notation are in order:

i) Substitution rules that are not scalar have to be introduced in matrix form. The substitution for $\{\Psi, A\}$ is a column vector $(\{\{\mathrm{a}\}\},\{\mathrm{b}\}\})$ and for $\{A, \Psi\}$ a row vector $(\{\{\mathrm{a}, \mathrm{b}\}\})$, corresponding to $\varphi_{A}$ being a scalar and $\varphi_{\Psi}$ being a doublet.

ii) The use of NonCommutativeMultiply (denoted by $* *$ ) when multiplying fields is mandatory, since these need to be treated as non-commuting objects during SuperTracer evaluation. 
iii) Whenever there are vector fields in the substitution rules, there needs to be an open Lorentz index matching that of the vector field. This Lorentz index has to be $\alpha$ [i] when $A$ is the first element, e.g. $\{A, \Psi\}$, and $\alpha[j]$ when $A$ is the second element, as in $\{\Psi, A\}$.

$i v$ ) We have also defined the heavy fermion masses with the third substitution rule. We have avoided using $\mathbf{M}$ for the mass, since this variable is already predefined in SuperTracer. Also, since the heavy fermion field is encoded in the $\varphi_{\Psi}=\left(\Psi \Psi^{c}\right)^{\top}$ doublet, a list with two elements is needed.

$v$ ) Finally, we have defined the action of the field-strength tensors on the fields. In this case, there is a single gauge group, the $\mathrm{U}(1)_{e}$, which we labeled with e, so we only need to specify the electric charges in the format \{e[charge] $\}$ for each of the fields. By default, the charges are assumed to be zero, which is why we input an empty list for $A$. A more complicated example with multiple gauge groups is given in the next section.

After substituting the EOM into the output for $\Psi$ (see (4.2)), one readily obtains ${ }^{7}$

$$
-\frac{i}{2} \mathrm{~S} \operatorname{Tr}\left\{\Delta_{\Psi} X_{\Psi A}^{[5 / 2]} \Delta_{A} X_{A \Psi}^{[5 / 2]}\right\}_{\text {hard }}=-\int \mathrm{d}^{d} x \frac{1}{16 \pi^{2}} 7 e^{2}\left(\frac{1}{2}+\log \frac{\bar{\mu}^{2}}{M_{\Psi}^{2}}\right) \frac{|y|^{2}}{M_{\Psi}^{2}}\left(\bar{\psi}_{L} \phi\right) i \not D\left(\phi \psi_{L}\right),
$$

where the parts highlighted in blue are kept implicit in SuperTracer for notational simplicity. The complete computation of the power-type Lagrangian is provided in the ancillary Mathematica notebook VLfermExample.nb. We have compared this result against an explicit computation done by diagrammatic matching, finding full agreement between the two. More details on this comparison are provided in appendix B.

We wish to close this section with a consequence of the $\gamma_{5}$ prescription employed in our approach. The supertrace

$$
\operatorname{STr}\left\{\Delta_{\Psi} X_{\Psi \psi}^{[1]} \Delta_{\psi} X_{\psi \Psi}^{[1]}\right\}
$$

contains terms with divergent loop integrals and odd numbers of $\gamma_{5}$. Due to the lack of a CP-violating interactions in the model, these terms cannot give rise to a contribution to the effective action of the form $F_{\mu \nu} \tilde{F}^{\mu \nu} \phi^{2}$. Indeed, in the SuperTracer calculation, a cancellation between the contributions from $\psi, \Psi$ and the ones from $\psi^{c}, \Psi^{c}$ takes place. This result is found in our prescription only if the traces are read from the correct starting point, which is guaranteed by construction in our formalism. At the diagrammatic level, a reading point ambiguity persists unless the diagrams are read in a consistent way. This means that some Dirac traces would have to be read against the conventional direction and interpreted as loops of charge-conjugated fermions instead of the usual way. We stress again that in our approach, the traces are automatically arranged in a way that fixed this issue. We have also checked against the diagrammatic computation that the $\mathrm{WC}$ of an

\footnotetext{
${ }^{7}$ As we show in the ancillary Mathematica notebook VLfermExample.nb, it is also possible to include the EOM for $\Psi$ in the input of STrTerm, yielding the same result.
} 
operator of the form $F_{\mu \nu} \tilde{F}^{\mu \nu} \phi_{1} \phi_{2}$, in a theory with two scalar fields instead of one, is correctly reproduced.

\section{$4.2 \quad S_{1}$ scalar leptoquark}

As our second example, we consider an $S_{1} \sim(\overline{\mathbf{3}}, \mathbf{1}, 1 / 3)$ scalar leptoquark extension of the SM, with the parenthesis indicating the $S_{1}$ representation under the SM gauge group $\mathrm{SU}(3)_{c} \times \mathrm{SU}(2)_{L} \times \mathrm{U}(1)_{Y}$. The Lagrangian of the model reads

$$
\begin{aligned}
\mathcal{L}= & \mathcal{L}_{\mathrm{SM}}+\left|D_{\mu} S_{1}\right|^{2}-M^{2}\left|S_{1}\right|^{2}-\left(\lambda_{1 L}^{i \alpha} \bar{q}_{i}^{c} \epsilon \ell_{\alpha} S_{1}+\lambda_{1 R}^{i \alpha} \bar{u}_{i}^{c} e_{\alpha} S_{1}+\text { h.c. }\right) \\
& -\frac{\lambda_{S}}{2}\left|S_{1}\right|^{4}-\lambda_{H S}|H|^{2}\left|S_{1}\right|^{2},
\end{aligned}
$$

where $\mathcal{L}_{\mathrm{SM}}$ is the SM Lagrangian, $\epsilon=i \sigma_{2}$ is the $\mathrm{SU}(2)_{L}$ anti-symmetric tensor, and $i$ and $\alpha$ are quark and lepton flavor indices, respectively. The covariant derivative acting on $S_{1}$ is given by

$$
D_{\mu} S_{1}=\left(\partial_{\mu}+i g_{c}\left(T^{a}\right)^{*} G_{\mu}^{a}-\frac{1}{3} i g_{Y} B_{\mu}\right) S_{1},
$$

with $T^{a}$ being the fundamental SU(3) generators, and $g_{c}$ and $g_{Y}$ the QCD and hypercharge gauge couplings, respectively. The complete one-loop matching conditions of this model to the SMEFT up to dimension-six operators can be found in [62]. Here, we do not intend to fully reproduce this result but rather to illustrate the one-loop matching procedure using the functional method described in section 2 and the SuperTracer package. First, we obtain the tree-level effective Lagrangian by substituting the EOM of $S_{1}$,

$$
S_{1}=\frac{1}{M^{2}}\left[\left(\lambda_{1 L}^{i \alpha}\right)^{*} \bar{\ell}_{\alpha} \epsilon q_{i}^{c}-\left(\lambda_{1 R}^{i \alpha}\right)^{*} \bar{e}_{\alpha} u_{i}^{c}\right]+\mathcal{O}\left(M^{-2}\right)
$$

into the Lagrangian, yielding

$$
\begin{aligned}
\mathcal{L}= & \mathcal{L}_{\mathrm{SM}}-\frac{1}{M^{2}} \lambda_{1 L}^{i \alpha}\left(\lambda_{1 L}^{j \beta}\right)^{*}\left(\bar{q}_{i}^{c} \epsilon \ell_{\alpha}\right)\left(\bar{\ell}_{\beta} \epsilon q_{j}^{c}\right)+\frac{1}{M^{2}} \lambda_{1 R}^{i \alpha}\left(\lambda_{1 R}^{j \beta}\right)^{*}\left(\bar{u}_{i}^{c} e_{\alpha}\right)\left(\bar{e}_{\beta} u_{j}^{c}\right) \\
& +\frac{1}{M^{2}}\left[\lambda_{1 L}^{i \alpha}\left(\lambda_{1 R}^{j \beta}\right)^{*}\left(\bar{q}_{i}^{c} \epsilon \ell_{\alpha}\right)\left(\bar{e}_{\beta} u_{j}^{c}\right)+\text { h.c. }\right)+\mathcal{O}\left(M^{-4}\right),
\end{aligned}
$$

which after applying Fierz transformations coincides with the tree-level Lagrangian in [62]. To perform the one-loop integration, we collect the fields into multiplets in the form of (2.33):

$$
\varphi_{S}=\left(\begin{array}{c}
S_{1} \\
S_{1}^{*}
\end{array}\right), \quad \varphi_{H}=\left(\begin{array}{c}
H \\
H^{*}
\end{array}\right), \quad \varphi_{f}=\left(\begin{array}{c}
f \\
f^{c}
\end{array}\right), \quad \varphi_{A}=A,
$$

with $A=B, W, G$ and $f=q, u, d, \ell, e$. As in the previous example, the $X$ terms for the $S_{1}$ part of the Lagrangian can be readily obtained from (2.35):

$$
\begin{aligned}
& X_{S A}^{[4,3]}=-\left(\begin{array}{c}
2 i Q_{S A}\left(D_{\mu} S_{1}\right) \\
-2 i Q_{S A}^{*}\left(D_{\mu} S_{1}\right)^{*}
\end{array}\right)-\left(\begin{array}{c}
Q_{S A} S_{1} \\
-Q_{S A}^{*} S_{1}^{*}
\end{array}\right) i D_{\mu}, \quad X_{A A^{\prime}}^{[6]}=-g_{\mu \nu} S_{1}^{\dagger}\left\{Q_{S A}, Q_{S A^{\prime}}\right\} S_{1}, \\
& X_{q S}^{[3 / 2]}=\left(\begin{array}{cc}
0 & \lambda_{1 L}^{*} \epsilon P_{R} \ell^{c} \\
\lambda_{1 L} \epsilon P_{L} \ell & 0
\end{array}\right), \quad X_{\ell S}^{[3 / 2]}=\left(\begin{array}{cc}
0 & -\lambda_{1 L}^{*} \epsilon P_{R} q^{c} \\
-\lambda_{1 L} \epsilon P_{L} q & 0
\end{array}\right),
\end{aligned}
$$




$$
\begin{array}{rlrl}
X_{u S}^{[3 / 2]} & =\left(\begin{array}{cc}
0 & \lambda_{1 R}^{*} P_{L} e^{c} \\
\lambda_{1 R} P_{R} e & 0
\end{array}\right), & X_{e S}^{[3 / 2]}=\left(\begin{array}{cc}
0 & \lambda_{1 R}^{*} P_{L} u^{c} \\
\lambda_{1 R} P_{R} u & 0
\end{array}\right), \\
X_{q l}^{[3]}=\left(\begin{array}{cc}
0 & \lambda_{1 L}^{*} \epsilon P_{R} S_{1}^{*} \\
\lambda_{1 L} \epsilon P_{L} S_{1} & 0
\end{array}\right), & X_{u e}^{[3]}=\left(\begin{array}{cc}
0 & \lambda_{1 R}^{*} P_{L} S_{1}^{*} \\
\lambda_{1 R} P_{R} S_{1} & 0
\end{array}\right), \\
X_{S H}^{[4]}=\lambda_{H S}\left(\begin{array}{cc}
S_{1} H^{\dagger} & S_{1} H^{\top} \\
S_{1}^{*} H^{\dagger} & S_{1}^{*} H^{\top}
\end{array}\right), & X_{H H}^{[6]}=\lambda_{H S}\left(\begin{array}{cc}
\left(S_{1}^{\dagger} S_{1}\right) \mathbb{1} & 0 \\
0 & \left(S_{1}^{\dagger} S_{1}\right) \mathbb{1}
\end{array}\right), \\
X_{S S}^{[2]}=\lambda_{H S}\left(\begin{array}{cc}
\left(H^{\dagger} H\right) \mathbb{1} & 0 \\
0 & \left(H^{\dagger} H\right) \mathbb{1}
\end{array}\right)+\lambda_{S}\left(\begin{array}{ccc}
\left(S_{1}^{\dagger} S_{1}\right) \mathbb{1}+S_{1} S_{1}^{\dagger} & S_{1} S_{1}^{\top} \\
S_{1}^{*} S_{1}^{\dagger} & \left(S_{1}^{\dagger} S_{1}\right) \mathbb{1}+S_{1} S_{1}^{\dagger}
\end{array}\right),
\end{array}
$$

with $Q_{S B}=g^{\prime} / 3, Q_{S W}=0$ and $Q_{S G}=-g_{s}\left(T^{a}\right)^{*}$. The corresponding $X$ terms for the $\mathrm{SM}$ interactions can be found e.g. in appendix B of [53]. As in the previous example, all the $X$ terms with permutated fields can be obtained by Hermitian conjugation of the ones above, that is $X_{B A}=\bar{X}_{A B}$. However, in contrast with the previous example, we now have an "open covariant derivative", i.e. a covariant derivative that does not act inside a commutator, in the $X_{S A}$ interaction. Following the prescription in (2.24), this means that $X_{A S}$ should be put in canonical form by making the derivative act from the rightmost, e.g.

$$
X_{A S}^{[4,3]}=\left(i\left(D_{\mu} S_{1}\right)^{\dagger} Q_{S A}-i\left(D_{\mu} S_{1}\right)^{\top} Q_{S A}^{*}\right)+\left(-S_{1}^{\dagger} Q_{S A} S_{1}^{\top} Q_{S A}^{*}\right) i D_{\mu} .
$$

We have once again included the mass dimension of the $X$ terms as a superscript. For the $X_{S A}$ and $X_{A S}$, which contain $X_{0}$ and $X_{1}^{\mu}$ terms in the expansion in (2.24), we have added two counting parameters instead of one, corresponding respectively to the term without open derivatives, $X_{0}$, and the term with one open derivative, $X_{1}^{\mu}$. The open derivative is not included in the counting of the $X$ mass dimensions. We emphasize that specifying $X$ mass dimensions in this way is useful to keep track of the EFT power counting.

Once the $X$ terms have been determined, we can proceed to the identification and evaluation of the relevant log-type and power-type supertraces yielding $\mathcal{L}_{\mathrm{EFT}}^{(1)}=\mathcal{L}_{\log }^{(1)}+$ $\mathcal{L}_{\text {power }}^{(1)}$. Once more, the log-type contribution can be readily obtained:

$$
\begin{aligned}
\mathcal{L}_{\log }^{(1)}= & \frac{1}{16 \pi^{2}}\left[-\frac{1}{12} \log \frac{\mu^{2}}{M^{2}} \operatorname{tr}_{G}\left\{F_{\mu \nu} F^{\mu \nu}\right\}-\frac{1}{60 M^{2}} \operatorname{tr}_{G}\left\{D_{\mu} F^{\mu \nu} D^{\rho} F_{\rho \nu}\right\}\right. \\
& \left.-\frac{1}{90 M^{2}} \operatorname{tr}_{G}\left\{i F_{\mu}{ }^{\nu} F_{\nu}{ }^{\rho} F_{\rho}{ }^{\mu}\right\}\right] \\
= & \frac{1}{16 \pi^{2}}\left[-\frac{g_{c}^{2}}{24} \log \frac{\mu^{2}}{M^{2}}\left(G_{\mu \nu}\right)^{a}\left(G^{\mu \nu}\right)^{a}-\frac{g_{Y}^{2}}{36} \log \frac{\mu^{2}}{M^{2}} B_{\mu \nu} B^{\mu \nu}-\frac{g_{c}^{2}}{120 M^{2}}\left(D_{\mu} G^{\mu \nu}\right)^{a}\left(D^{\rho} G_{\rho \nu}\right)^{a}\right. \\
& \left.-\frac{g_{Y}^{2}}{180 M^{2}} \partial_{\mu} B^{\mu \nu} \partial^{\rho} B_{\rho \nu}+\frac{g_{c}^{3}}{360 M^{2}} f_{a b c}\left(G_{\mu}{ }^{\nu}\right)^{a}\left(G_{\nu}{ }^{\rho}\right)^{b}\left(G_{\rho}{ }^{\mu}\right)^{c}\right]
\end{aligned}
$$

where we took $F_{\mu \nu}=g_{c} T^{a}\left(G_{\mu \nu}\right)^{a}+g_{Y} Y_{S_{1}} B_{\mu \nu}$ in the second equality. Note the implicit color factor arising from the gauge trace in the terms with $B_{\mu \nu}$. This result coincides with the one in [62]. The same expression for the first equality in (4.18) is obtained by SuperTracer by running 
Out[12] $=\quad-\frac{1}{24} \log \left[\frac{\bar{\mu}^{2}}{\mathrm{M}_{\mathrm{H}}^{2}}\right] \mathrm{G}^{\mu \nu} * * \mathrm{G}^{\mu \nu}+\frac{1}{120} \frac{1}{\mathrm{M}_{\mathrm{H}}^{2}} \mathrm{D}_{\mu} \mathrm{G}^{\mu \nu} * * \mathrm{D}_{\rho} \mathrm{G}^{\nu \rho}-\frac{1}{180} \mathrm{i} \frac{1}{\mathrm{M}_{\mathrm{H}}^{2}} \mathrm{G}^{\mu \nu} * * \mathrm{G}^{\mu \rho} * * \mathrm{G}^{\nu \rho}$

and accounting for the doubling of contributions since $S_{1}$ is a complex scalar field, and the contributions from both $S_{1}$ and $S_{1}^{*}$ should be included. As for the power terms, the first thing to note is that $X_{A A}, X_{S H}$ and $X_{H H}$ do not contribute at mass dimension six due to their high mass dimension. Since we do not intend to perform the full matching procedure, but just to illustrate the method in a more realistic example, we set $\lambda_{1 R}$ to zero and neglect the SM Yukawa couplings. In this case, the only relevant SM $X$ terms are $X_{\psi A}^{[3 / 2]}$ and their conjugates. Following the prescription in section 2.4, we collect all fields of the same type into multiplets, such that e.g. $\varphi_{A}=(G W B)^{\top}$ and $X_{\Phi A}=\left(X_{S G} 0 S_{S B}\right)$. The Lagrangian for the power terms then reads

$$
\begin{aligned}
\mathcal{L}_{\text {power }}^{(1)}=- & \frac{i}{2}\left[\operatorname{STr}\left\{\Delta_{\Phi} X_{\Phi \Phi}^{[2]}\right\}+\mathrm{S} \operatorname{Tr}\left\{\Delta_{\Phi} X_{\Phi A}^{[4,3]} \Delta_{A} X_{A \Phi}^{[4,3]}\right\}+\frac{1}{2} \mathrm{~S} \operatorname{Tr}\left\{\left(\Delta_{\Phi} X_{\Phi \Phi}^{[2]}\right)^{2}\right\}\right. \\
& +\mathrm{S} \operatorname{Tr}\left\{\Delta_{\Phi} X_{\Phi \psi}^{[3 / 2]} \Delta_{\psi} X_{\psi \Phi}^{[3 / 2]}\right\}+\frac{1}{3} \mathrm{~S} \operatorname{Tr}\left\{\left(\Delta_{\Phi} X_{\Phi \Phi}^{[2]}\right)^{3}\right\} \\
& +\mathrm{S} \operatorname{Tr}\left\{\Delta_{\Phi} X_{\Phi \Phi}^{[2]} \Delta_{\Phi} X_{\Phi \psi}^{[3 / 2]} \Delta_{\psi} X_{\psi \Phi}^{[3 / 2]}\right\}+\left(\operatorname{STr}\left\{\Delta_{\Phi} X_{\Phi A}^{[4,3]} \Delta_{A} X_{A \psi}^{[3 / 2]} \Delta_{\psi} X_{\psi \Phi}^{[3 / 2]}\right\}+\text { h.c. }\right) \\
& +\mathrm{S} \operatorname{Tr}\left\{\Delta_{\Phi} X_{\Phi \psi}^{[3 / 2]} \Delta_{\psi} X_{\psi \psi}^{[3]} \Delta_{\psi} X_{\psi \Phi}^{[3 / 2]}\right\}+\operatorname{STr}\left\{\Delta_{\Phi} X_{\Phi \psi}^{[3 / 2]} \Delta_{\psi} X_{\psi A}^{[3 / 2]} \Delta_{A} X_{A \psi}^{[3 / 2]} \Delta_{\psi} X_{\psi \Phi}^{[3 / 2]}\right\} \\
& \left.+\frac{1}{2} \operatorname{STr}\left\{\left(\Delta_{\Phi} X_{\Phi \psi}^{[3 / 2]} \Delta_{\psi} X_{\psi \Phi}^{[3 / 2]}\right)^{2}\right\}\right]_{\text {hard }} .
\end{aligned}
$$

Once more, note the symmetry factors $1 / 2$ and $1 / 3$ in some of the traces. This result is reproduced by the program from the input

$$
\begin{aligned}
& \operatorname{In}[13]:=\mathrm{Xterms}=\{\mathrm{X}[\{\Phi, \mathrm{A}\},\{4,3\}], \mathrm{X}[\{\psi, \Phi\}, 3 / 2], \mathrm{X}[\{\psi, \psi\}, 3], \\
& \mathrm{X}[\{\Phi, \Phi\}, 2], \mathrm{X}[\{\psi, \mathrm{A}\}, 3 / 2]\} \text {; } \\
& \text { LagPower }=\text { PowerTerms }[\text { Xterms }]
\end{aligned}
$$

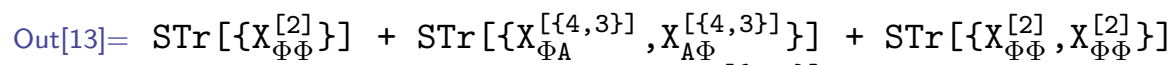

$$
\begin{aligned}
& +\operatorname{STr}\left[\left\{\mathrm{X}_{\Phi \psi}^{[3 / 2]}, \mathrm{X}_{\psi \Phi}^{[3 / 2]}\right\}\right]+\operatorname{STr}\left[\left\{\mathrm{X}_{\Phi \mathrm{A}}^{[\{4,3\}]}, \mathrm{X}_{\mathrm{A} \psi}^{[3 / 2]}, \mathrm{X}_{\psi \Phi}^{[3 / 2]}\right\}\right] \\
& +\operatorname{STr}\left[\left\{\mathrm{X}_{\Phi \Phi}^{[2]}, \mathrm{X}_{\Phi \Phi}^{[2]}, \mathrm{X}_{\Phi \Phi}^{[2]}\right\}\right]+\operatorname{STr}\left[\left\{\mathrm{X}_{\Phi \Phi}^{[2]}, \mathrm{X}_{\Phi \psi}^{[3 / 2]}, \mathrm{X}_{\psi \Phi}^{[3 / 2]}\right\}\right] \\
& +\operatorname{STr}\left[\left\{\mathrm{X}_{\Phi \psi}^{[3 / 2]}, \mathrm{X}_{\psi \mathrm{A}}^{[3 / 2]}, \mathrm{X}_{\mathrm{A} \Phi}^{[\{4,3\}]}\right\}\right]+\operatorname{STr}\left[\left\{\mathrm{X}_{\Phi \psi}^{[3 / 2]}, \mathrm{X}_{\psi \psi}^{[3]}, \mathrm{X}_{\psi \Phi}^{[3 / 2]}\right\}\right] \\
& +\operatorname{STr}\left[\left\{\mathrm{X}_{\Phi \psi}^{[3 / 2]}, \mathrm{X}_{\psi \mathrm{A}}^{[3 / 2]}, \mathrm{X}_{\mathrm{A} \psi}^{[3 / 2]}, \mathrm{X}_{\psi \Phi}^{[3 / 2]}\right\}\right]+\operatorname{STr}\left[\left\{\mathrm{X}_{\Phi \psi}^{[3 / 2]}, \mathrm{X}_{\psi \Phi}^{[3 / 2]}, \mathrm{X}_{\Phi \psi}^{[3 / 2]}, \mathrm{X}_{\psi \Phi}^{[3 / 2]}\right\}\right]
\end{aligned}
$$

where, we remind the reader, the symmetry and the $-i / 2$ factors are taken as part of the definition of STr in SuperTracer. As an example, we show the evaluation of the second term, corresponding in the diagrammatic language to a one-loop gauge correction to the propagator of the $S_{1}$ leptoquark. As usual, first we have to define the fields entering in the $X$ substitutions using AddField, which in this case is just the $S_{1}$ leptoquark:

$\ln [14]:=$ AddField $[S 1, \Phi, \mathrm{Y}[1 / 3]]$ 
where we labeled hypercharge by Y. Once this has been done, we can input our $X$ substitution in (4.16) into STrTerm to obtain desired result:

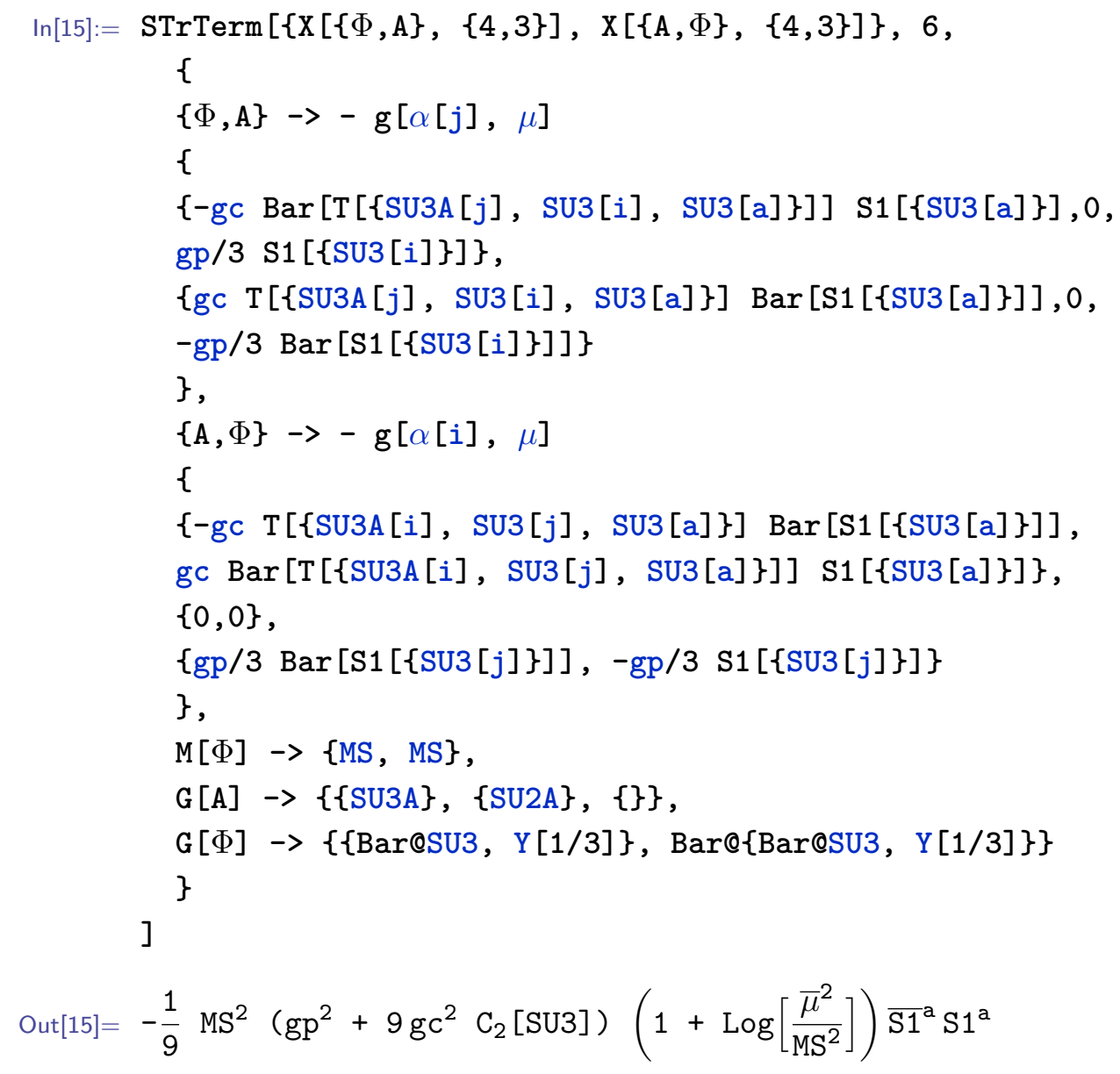

Since we are introducing field substitutions with "open covariant derivatives" and with gauge indices, some comments on the notation of the input are in order:

i) As previously mentioned, the $X_{S A}^{[4,3]}$ and $X_{A S}^{[4,3]}$ interactions in (4.16) and (4.17) contain terms with one open covariant derivative. At present, SuperTracer only supports $X$ substitutions for terms with up to two open covariant derivatives, namely for $X_{0}$, $X_{1}^{\mu}$ and $X_{2}^{\mu \nu}$ in the expansion in (2.24). When providing $X$ substitutions, a separate rule for any relevant $X_{0}, X_{1}^{\mu}$ and/or $X_{2}^{\mu \nu}$ must be added. They all start with the replacement rule $\{$ FieldType1, FieldType2\}-> . . but they are differentiated by the open indices in the rule: no open index for $X_{0}$ (as we did with all substitutions so far), $\mu$ for $X_{1}^{\mu}$, and $\mu$ and $\nu$ for $X_{2}^{\mu \nu}$. In the present example, only the $X_{1}^{\mu}$ part of $X_{S A}^{[4,3]}$ and $X_{A S}^{[4,3]}$ contribute at dimension six, as trivially seen by adding the $X$ term dimensions in the supertrace of our example. Hence, we only need to input a replacement rule for this term. Indeed, the two substitutions in the example above contain an open $\mu$ index in $\mathrm{g}[\alpha[j], \mu]$. The symbols used for entering open Lorentz indices in the substitution must always be $\mu$ in the case of one open index or $\mu$ and $\nu$ for two open indices. 
ii) The input of gauge indices in $X$ substitutions requires a representation label defining the kind of index, specified as rep [index]. In our example, we chose SU3A and SU3 to distinguish adjoint and fundamental $\mathrm{SU}(3)_{c}$ indices, respectively, although any label names preferred by the user are equally valid. As in the vector-like fermion example, the action of $G_{\mu \nu}$ on each field needs to be defined by the appropriate substitution rules, the ones for $\mathrm{G}[\mathrm{A}]$ and $\mathrm{G}[\Phi]$ in our example. For instance, the substitution rule $\mathrm{G}[\Phi] \rightarrow\{$ Bar@SU3, Y $[1 / 3]\}, \operatorname{Bar} @\{\mathrm{Bar} @ S U 3, \mathrm{Y}[1 / 3]\}\}$ indicates that $S_{1}$ transforms in the antifundamental of $\mathrm{SU}(3)_{c}$ (since we consider SU3 to denote fundamental $\mathrm{SU}(3)_{c}$ indices and Bar gives the conjugate) and has hypercharge 1/3 (labeled by $\mathrm{Y}$ ), while $S_{1}^{*}$ transforms in the fundamental of $\mathrm{SU}(3)_{c}$ and has hypercharge $-1 / 3$. In the output, all indices are displayed in the same manner as superindices of the fields (and couplings) since tracking their type is straightforward in most cases. When this is not the case, these can be made explicit by evaluating the command ShowRep [True], which globally turns on the printing of index types. This behavior can be deactivated again by using ShowRep[False]. Also looking into the InputForm/FullForm yields the information about the index types.

iii) The open indices in the $X$ substitutions are all identified to the program by always giving them the same index name. For the substitution of $X_{\eta_{1} \eta_{2}}$ all the open indices from the $\eta_{1}$ field, must be given in the form rep [i], whereas the open indices from $\eta_{2}$ must be entered as rep $[j]$. In both cases rep can freely be chosen to fit the index type, but the names must always be $i$ and $j$, respectively. For the indices contracted internally in $X$, there are no rules as to the index names. The usage of open indices is demonstrated in the example substitution, where the reader can see the use of $i$ and $j$ in the open SU3 and SU3A indices. We add that it is unproblematic to use the same index name multiple times in this context as e.g. rep1 [i] and rep2 [i] are recognized as different indices by SuperTracer.

iv) Gauge generators $T_{i j}^{a}$ are specified in SuperTracer as T [ $\left.\{r e p A[a], r e p R[i], r e p R[j]\}\right]$. These objects present some basic properties such as being traceless $T_{i i}^{a}=0$ and Hermitian $\bar{T}_{i j}^{a}=T_{j i}^{a}$. The relation $T_{i k}^{a} T_{k j}^{a}=C_{2}(R) \delta_{i j}$, where $C_{2}(R)$ is the quadratic Casimir of the representation with $i, j$ indices, is also encoded in $\mathrm{T}$, along with $T_{i j}^{a} T_{j i}^{b}=S_{2}(R) \delta^{a b}$ for the Dynkin index $S_{2}(R)$. Other group specific properties can be added on a case-by-case basis by the user, as we show in the example notebook S1LQExample.nb.

$v$ ) Finally, it is worth noting that other indices than gauge can be included in the $X$ substitutions. By default SuperTracer will assume all field indices are gauge when finding the action of field-strength tensors. One can, however, assign index labels to be treated as global or flavor indices by calling AddGlobalSym [rep], after which all indices with the label rep will be treated as global indices. ${ }^{8}$ Flavor is a predefined global index, and if e.g. we were to give $S_{1}$ a flavor index, we would call it with S1 [\{SU3 [a], Flavor [b] \}]. In this case, we should also make sure to account for the flavor index being contracted along $S_{1}$ propagators, which can be accounted for

\footnotetext{
${ }^{8}$ The list of global labels can be reset with ResetGlobalSym [] .
} 
by providing the global labels when we set the action of the field-strength tensors on the field: $G[\Phi] \rightarrow\{$ Bar@SU3, Y [1/3], Flavor\}, Bar@\{Bar@SU3, Y[1/3], Flavor\}\}. While we do not demonstrate global indices in action here, the S1LQExample.nb notebook example provides an example of this functionality.

More examples, including EOM substitutions for $S_{1}$ and some group algebra simplifications, are provided in the S1LQExample.nb notebook example.

As noted before, the output from SuperTracer can be directly compared to the results found in ref. [62]. Comparing the full operator basis requires a significant amount of manipulations of the results due to the lack of an automatic Fierz transformation routine. However, we have done partial checks and find agreement with the expressions we checked with a single exception. ${ }^{9}$

\section{Conclusions}

Computing a low-energy effective Lagrangian from a given theory is a common and exceedingly mechanical task at the beginning of most studies both in and beyond the SM. Having an automated solution greatly simplifies and accelerates these initial stages and puts matching calculations into the realm of something that can be quickly realized to test ideas without having to devote large amounts of time to it. In many cases, the more interesting phenomenology arises at the loop-level, for example when studying flavor physics. Therefore, an automated solution should be able to include at least the one-loop effects.

SuperTracer is an important step in this direction. It allows for the computation of functional supertraces, which is the central part of a functional matching computation, in an automated fashion. While the diagrammatic approach to matching is arguably more common, the path integral formalism holds several advantages. First and foremost, it requires no knowledge of the operator basis, circumventing the risk of missing an operator. Secondly, the formalism lends itself incredibly well to automation, something that cannot be said for the diagrammatic approach: finding an operator basis and then constructing and computing all contributing Feynman graphs to fix their matching coefficients is a disproportionately more complicated task to automate. Furthermore, computing the necessary prerequisites for the functional computations is almost trivial. The labor-intensive task of performing the momentum expansion and the actual computation of the one-loop effective action is then mostly done by SuperTracer.

At the current stage, performing a one-loop matching computation with SuperTracer still requires a significant amount of human intervention. While it is true that computing the ingredients is easy, inputting them into the program is still somewhat time-consuming and requires a certain amount of care. Furthermore, the output produces an effective Lagrangian that typically needs to be manipulated to become useful in an actual physics computation. First, SuperTracer does not provide the interaction terms $X$ or the EOMs of the heavy fields, which need to be provided by the user. For this, the program would need to know the full theory Lagrangian and derive these expressions from its functional

\footnotetext{
${ }^{9}$ We believe the authors of ref. [62] are missing a factor of two in their matching coefficients $\mathcal{C}_{H B}$ and $\mathcal{C}_{H G}$.
} 
derivatives. Second, the resulting effective Lagrangian contains redundant operators, which can be reduced by the standard methods such as reduction of products of Dirac matrices, integration-by-parts identities, field redefinitions and Fierz transformations. These shortcomings will be addressed in the upcoming release of a Mathematica package called MATCHETE [61], which will contain SuperTracer at its heart. MATCHETE will allow the user to input a Lagrangian and specify the power-counting rules of the fields. The program will then automatically compute the one-loop EFT Lagrangian in a minimal basis.

Already in its current form, without the expected benefits from the full release of MATCHETE, the program presented here provides a tremendous simplification to a one-loop matching computations, paving the way for a fully automated solution. While it often remains illuminating to perform parts of these computations manually, a computer program can provide valuable cross-checks. We believe however, that the outlook of fully relegating the drawn-out task of a matching computation to a machine has exciting implications for model building and phenomenology in the future.

\section{Acknowledgments}

We are grateful to Timothy Cohen, Xiaochuan Lu, and Zhengkang Zhang for communications about their related work [54], for providing cross-checks for our program and for coordinating the release date of their package with ours. The work of J.F. was supported by the Cluster of Excellence 'Precision Physics, Fundamental Interactions, and Structure of Matter (PRISMA+ EXC 2118/1) funded by the German Research Foundation (DFG) within the German Excellence Strategy (Project ID 39083149). J.P. and F.W. have received funding from the European Research Council (ERC) under the European Union's Horizon 2020 research and innovation program under grant agreement 833280 (FLAY). J.P., A.E.T., and F.W. have received funding by the Swiss National Science Foundation (SNF) under contract 200021-175940. The work of A.E.T. has received funding from the Swiss National Science Foundation (SNF) through the Eccellenza Professorial Fellowship "Flavor Physics at the High Energy Frontier" project number 186866.

\section{A Special SuperTracer variables}

For completeness, table 3 provides a list of all public SuperTracer variables that were not described in section 3. These variables are used internally in SuperTracer outputs, as can be seen explicitly by applying to them the Mathematica routine InputForm/FullForm. While knowing the internal representation of symbols is useful for further manipulations of SuperTracer outputs, the output is formatted to make it as intuitive as possible for the user.

\section{B Diagrammatic matching for the vector-like fermion example}

In this appendix we shall further outline the comparison between the diagrammatic and the functional matching calculation in section 4.1. To compare the results swiftly, we choose 


\begin{tabular}{|c|c|}
\hline Internal notation & Description \\
\hline LTerm [cof, op $]$ & $\begin{array}{l}\text { Denotes a Lagrangian operator op with co- } \\
\text { efficient cof. }\end{array}$ \\
\hline $1 i[$ seq] & Denotes a sequence seq of Lorentz indices. \\
\hline DiracProduct [seq] & $\begin{array}{l}\text { Represents the product of Dirac matrices, } \\
\text { charge conjugation matrices and chiral pro- } \\
\text { jectors in the sequence seq. Argument } \\
l_{i}[\mu] \text { is used for } \gamma_{\mu}, l_{i}[\mu, \ldots] \text { for } \Gamma_{\mu, \ldots}^{(n)} \text {, } \\
\text { and } 5 \text { for } \gamma_{5} \text {. The DiracProduct head is not } \\
\text { show in the standard output. }\end{array}$ \\
\hline $\operatorname{Proj}[ \pm 1]$ & $\begin{array}{l}\text { Chiral projector }\left(+1 \text { for } P_{R} \text { and }-1 \text { for } P_{L}\right) \text {. } \\
\text { It can only be used inside DiracProduct. Its } \\
\text { output has the same special form as PL, PR. }\end{array}$ \\
\hline $\operatorname{CovD}[1 \mathrm{i}[<$ seq1 $1>], \operatorname{expr}, 1 i[<$ seq2 $]]$ & $\begin{array}{l}\text { Internal representation of a sequence of } \\
\text { covariant derivatives, given by the op- } \\
\text { tional argument <seq1>, acting of the ex- } \\
\text { pression expr. The expression can have } \\
\text { Lorentz indices, given by the optional se- } \\
\text { quence <seq2>. This variable has a special } \\
\text { output format, e.g. CovD }[1 i[\mu], f, 1 i[]] \\
\text { shows as } \mathrm{D}_{\mu} \mathrm{f} \text { and } \operatorname{CovD}[\operatorname{li}[\mu, \nu], \mathrm{f}, \mathrm{li}[\mu]] \\
\text { as } \mathrm{D}_{\mu} \mathrm{D}_{\nu} \mathrm{f}^{\mu} \text {. }\end{array}$ \\
\hline $\begin{array}{l}\text { CovD }[1 i[<\text { seq }>\text {, Field [label, type, } \\
\text { indices, charge], li []] }\end{array}$ & $\begin{array}{l}\text { Internal representation of a field and covari- } \\
\text { ant derivatives acting on it. The optional } \\
\text { argument <seq is a sequence of Lorentz } \\
\text { indices marking the covariant derivatives, } \\
\text { label is the name of the field, type the field } \\
\text { type (see table } 1 \text { ), indices a list of field in- } \\
\text { dices, and charge a number (or a list of num- } \\
\text { bers) indicating the field charge(s) under the } \\
\text { Abelian group(s). }\end{array}$ \\
\hline $\operatorname{CovD}[1 \mathrm{i}[\langle$ seq $>], \mathrm{G}[$ gauge $], 1 \mathrm{i}[\mu, \nu]]$ & $\begin{array}{l}\text { Internal representation of a field-strength } \\
\text { tensor, and covariant derivatives acting on } \\
\text { it. The optional argument <seq }>\text { is a se- } \\
\text { quence of Lorentz indices marking the co- } \\
\text { variant derivatives. The argument gauge in- } \\
\text { dicates the gauge structure and can be either } \\
\text { a symbol labeling the Abelian symmetry, or } \\
\text { two non-Abelian indices in the form }\{\mathrm{a}, \mathrm{b}\} \text {. } \\
\text { The arguments } \mu \text { and } \nu \text { are the Lorentz in- } \\
\text { dices of the field-strength tensor. }\end{array}$ \\
\hline
\end{tabular}




\begin{tabular}{|c|c|}
\hline Internal notation & Description \\
\hline Index [index, rep] & $\begin{array}{l}\text { A header to distinguish internally that a } \\
\text { certain index is an index variable and } \\
\text { belongs to the representation rep. In- } \\
\text { dices entered in other routines in the form } \\
\text { rep [index] are internally transformed to } \\
\text { Index [index, rep]. For the output, in- } \\
\text { dices are displayed as superscripts and by } \\
\text { default their representation is not printed. } \\
\text { The routine ShowRep [True] can be evalu- } \\
\text { ated to globally activate displaying indices } \\
\text { with their representation as subscript. The } \\
\text { stardard behaviour is recovered by evaluat- } \\
\text { ing ShowRep[False]. }\end{array}$ \\
\hline $\operatorname{Dim}[r e p]$ & $\begin{array}{l}\text { Dimension of the representation rep. This } \\
\text { variable has the special output format } \mathrm{N}_{\text {rep }} \text {. }\end{array}$ \\
\hline $\mathrm{S} 2 \mathrm{R}[\mathrm{rep}]$ & $\begin{array}{l}\text { Dynkin index of representation rep. This } \\
\text { variable has the special output format } \\
\mathrm{S}_{2} \text { [rep]. }\end{array}$ \\
\hline $\mathrm{C} 2 \mathrm{R}[\mathrm{rep}]$ & $\begin{array}{l}\text { Quadratic Casimir of representation rep. } \\
\text { This variable has the special output format } \\
\mathrm{C}_{2} \text { [rep]. }\end{array}$ \\
\hline AddGlobalSym [rep] & $\begin{array}{l}\text { Adds the representation rep to the list of } \\
\text { global symmetry representations (except for } \\
\text { Flavor, all representations are local by de- } \\
\text { fault). The routine ResetGlobalSym [] can } \\
\text { be used to reset the list of global symmetry } \\
\text { representations to \{Flavor\}. }\end{array}$ \\
\hline CanonizeIndices [expr] & $\begin{array}{l}\text { Brings the Lorentz indices in expr to canon- } \\
\text { ical order by attempting different index } \\
\text { relabelings. This routine is called by } \\
\text { SuperSimplify. }\end{array}$ \\
\hline SimplifyOutput [expr] & $\begin{array}{l}\text { Applies integration by parts, commutator, } \\
\text { and Jacobi identities to match expr to a ba- } \\
\text { sis of operators. This routine is called by } \\
\text { SuperSimplify. }\end{array}$ \\
\hline
\end{tabular}

Table 3. SuperTracer notation relevant for output manipulations.

a basis for the effective Lagrangian closest to the output of SuperTracer, which can be found in the ancillary Mathematica notebook VLfermExample.nb. We then compute an exhaustive set of $n$-point functions to fix the couplings of the effective theory. 
Note that in the diagrammatic approach, several $n$-point functions can match to the same effective operators since one distinguishes between amplitudes with and without extra gauge fields. As an example, consider an effective operator of the form

$$
\bar{\psi} \phi i \not D \phi \psi \text {. }
$$

This operator contributes to both a four-point function $\psi^{2} \phi^{2}$ as well as a five-point function with an additional gauge field. Herein lies one of the advantages of the covariant functional approach, as it computes the WC of this operator directly. On the other hand, the relation of the two amplitudes by gauge invariance serves as a valuable cross-check of the diagrammatic calculation.

Integrating out the heavy vector-like fermion $\Psi$ produces two types of matching corrections: first, we obtain hard corrections to the renormalizable interactions of the soft fields in the theory, including the ones that were not present in the UV theory. We thus split the low-energy Lagrangian into a leading-power and subleading-power piece, defining

$$
\mathcal{L}_{\mathrm{EFT}}=\sum_{i} c_{i} o_{i}
$$

At leading power, we have the one-loop effective Lagrangian:

$$
\left.\mathcal{L}_{\mathrm{EFT}}^{(1)}\right|_{M^{0}}=\frac{c_{\phi}}{2}\left(\partial_{\mu} \phi\right)\left(\partial^{\mu} \phi\right)-\frac{c_{m}}{2} \phi^{2}-\frac{c_{\lambda}}{4 !} \phi^{4}-\frac{c_{A}}{4} F_{\mu \nu} F^{\mu \nu}+c_{\psi} \bar{\psi}_{L}(i \not D) \psi_{L} .
$$

Note that non-vanishing matching coefficients $c_{\phi}, c_{A}$ and $c_{\psi}$ imply that the light degrees of freedom are not canonically normalized. The presentation was chosen this way to emulate the output of the functional calculation more closely. In a diagrammatic computation, one would usually assume canonically normalized light fields and include hard wave-function corrections using the LSZ formula. Since the functional trace corresponds to 1PI diagrams, such corrections are not included in the matching coefficients. Instead, we treat $o_{\phi}, o_{m}$, $o_{A}$ and $o_{\psi}$ as independent composite operators and match them to the hard regions of the two-point functions. The result with canonically normalized fields can be recovered by performing the field redefinitions:

$$
\phi \rightarrow \frac{\phi}{\sqrt{1+c_{\phi}}}, \quad \quad A_{\mu} \rightarrow \frac{A_{\mu}}{\sqrt{1+c_{A}}}, \quad \psi \rightarrow \frac{\psi}{\sqrt{1+c_{\psi}}} .
$$

At dimension six, we define the following effective Lagrangian, this time including both the dimension-six terms from the tree-level and one-loop Lagrangian:

$$
\begin{aligned}
\left.\mathcal{L}_{\mathrm{EFT}}\right|_{M^{2}}= & \frac{c_{1}}{2 M^{2}} \phi D^{4} \phi+\frac{c_{2}}{4 M^{2}} F_{\mu \nu} \partial^{2} F^{\mu \nu}-\frac{i c_{3}}{2 M^{2}} \bar{\psi}\left\{\not D, D^{2}\right\} P_{L} \psi \\
& +\frac{c_{4} e}{M^{2}} F_{\nu \rho} \bar{\psi} \Gamma^{\mu \nu \rho} P_{L} D_{\mu} \psi+\frac{c_{5} e}{M^{2}}\left(\partial_{\nu} F^{\mu \nu}\right) \bar{\psi} \gamma_{\mu} P_{L} \psi \\
& +\frac{c_{6}}{2 M^{2}} \bar{\psi} \phi i \not D \phi P_{L} \psi+\frac{c_{7}}{4 ! M^{2}} \phi^{2} D^{2} \phi^{2}-\frac{c_{8}}{8 M^{2}} F_{\mu \nu} F^{\mu \nu} \phi^{2}+\frac{c_{9}}{6 ! M^{2}} \phi^{6},
\end{aligned}
$$

where $\Gamma^{\mu \nu \rho}=\gamma^{[\mu} \gamma^{\nu} \gamma^{\rho]}$. The way the Lagrangian is written, we have anticipated the coefficients $c_{i}$ to be real in the matching example we are considering. The first line of 
this Lagrangian generates power-corrections to the propagators and, in the case of charged fields, power corrections to the gauge couplings. Note that the most general basis should also include the $\mathrm{CP}$-odd counterpart of $o_{8}$, with one field-strength tensor replaced by its dual. Due to the absence of a source of CP-violation in the UV model, we drop it right away.

To demonstrate the matching procedure, let us begin with the example of the two-point functions. Up to second order in the expansion in $p^{2} / M^{2}$ (with $p^{\mu}$ being the momentum of the field), these are:

$$
\begin{aligned}
& \phi \\
& \phi
\end{aligned}=\frac{i \alpha_{y}}{2 \pi}\left\{p^{2}\left(\Delta_{\mu}+\frac{1}{2}\right)-2 M^{2}\left(\Delta_{\mu}+1\right)+\frac{p^{4}}{3 M^{2}}\right\},
$$

with $\Delta_{\mu}=1 / \epsilon+\log \mu^{2} / M^{2}, \alpha_{y}=|y|^{2} / 4 \pi, \alpha=e^{2} / 4 \pi$ and $d=4-2 \epsilon$. The above expressions

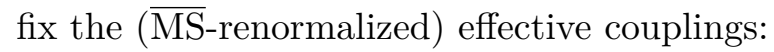

$$
\begin{aligned}
c_{\phi} & =\frac{\alpha_{y}}{2 \pi}\left(\log \frac{\mu^{2}}{M^{2}}+\frac{1}{2}\right), & c_{m} & =\frac{\alpha_{y}}{\pi} M^{2}\left(\log \frac{\mu^{2}}{M^{2}}+1\right), \\
c_{A} & =\frac{\alpha}{3 \pi} \log \frac{\mu^{2}}{M^{2}}, & c_{2} & =\frac{\alpha}{15 \pi}, \\
c_{\psi} & =\frac{\alpha_{y}}{8 \pi}\left(\log \frac{\mu^{2}}{M^{2}}+\frac{\alpha_{y}}{2}\right), & c_{3} & =\frac{\alpha_{y}}{12 \pi} .
\end{aligned}
$$

Inserting these results into the Lagrangian (B.3) and the first line of (B.5) reproduces the output from the program when evaluating the following log-type and power-type supertraces

$$
\operatorname{STr}\left\{\Delta_{\Psi} X_{\Psi \psi}^{[1]} \Delta_{\psi} X_{\psi \Psi}^{[1]}\right\}, \quad \operatorname{STr} \ln \Delta_{\Psi}, \quad \operatorname{STr}\left\{\Delta_{\Psi} X_{\Psi \phi}^{[3 / 2]} \Delta_{\phi} X_{\phi \Psi}^{[3 / 2]}\right\} .
$$

The operator $o_{3}$ is the first one in the sequence that contributes to more than one amplitude, as it is possible to contract it with up to three external photon states. Naturally, the next step is to compute the three-point function of two fermions and one gauge field. It is found to be

$$
\begin{aligned}
& \underbrace{\psi}_{\psi} \phi=\frac{i e \alpha_{y}}{8 \pi}\left\{\gamma^{\mu} P_{L}\left[\Delta_{\mu}+\frac{3}{2}\right]+\frac{1}{M^{2}}\left[\gamma^{\mu} P_{L}\left(\frac{11\left(k_{1}^{2}+k_{2}^{2}\right)+7 k_{1} \cdot k_{2}}{9}\right)\right.\right. \\
& \left.\left.-\frac{p^{\mu}}{6} \not p P_{L}-\not k_{1} \gamma^{\mu} \not k_{2} P_{L}\right]\right\} \text {, }
\end{aligned}
$$



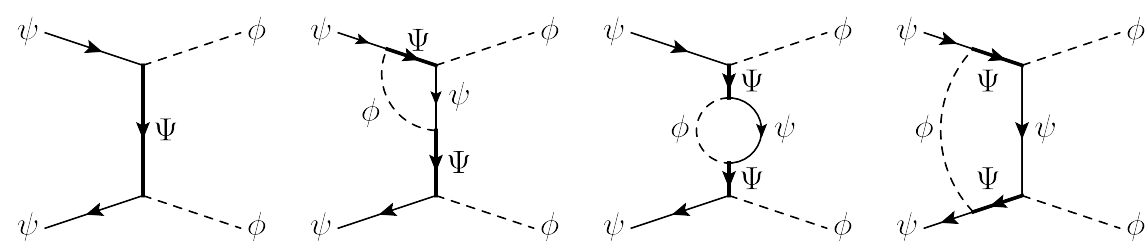

Figure 2. Example Feynman diagrams contributing to the one-loop matching up to $\mathcal{O}\left(\alpha_{y}^{2}\right)$ of the effective operator $o_{6}$.

where the fermion and anti-fermion have outgoing momenta $k_{1}$ and $k_{2}$, respectively, and we define $p^{\mu}=k_{1}^{\mu}-k_{2}^{\mu}$. One recognizes immediately that the first term is reproduced by $o_{\psi}$ with the matching condition found from the two-point function of the fermion. While in the diagrammatic calculation this is a sanity check, it never occurs in the functional calculation since the supertrace

$$
\operatorname{STr}\left\{\Delta_{\Psi} X_{\Psi \phi}^{[3 / 2]} \Delta_{\phi} X_{\phi \Psi}^{[3 / 2]}\right\}
$$

immediately gives rise to the operator $o_{\psi}$, generating both amplitudes. The beauty of the functional calculation shines even brighter in the subleading-power contributions. For the diagrammatic matching, one needs to first find the appropriate operator basis, derive the corresponding amplitudes, and match them to the expression above. Needless to say, this is a rather tedious exercise. On the other hand, the effective interactions are all immediately found by evaluating a single supertrace. From the diagrammatic computation we find:

$$
c_{4}=-\frac{\alpha_{y}}{16 \pi}, \quad c_{5}=\frac{\alpha_{y}}{9 \pi},
$$

which matches the output from SuperTracer perfectly.

The last example that deserves attention is the matching to the operator $o_{6}$. This is the first (and only) operator in $\mathcal{L}_{\mathrm{EFT}}^{(1)}$ that is also generated at tree-level. In the diagrammatic approach, we evaluate all Feynman graphs corresponding to the amplitudes up to the desired order in the couplings. We find that the one-loop corrections proportional to $\mathcal{O}(\alpha)$ vanish in the sum over all diagrams. The non-vanishing contributions are then found from graphs akin to those shown in figure 2. They lead us to the matching condition:

$$
c_{6}=2|y|^{2}\left[1-\frac{\alpha_{y}}{2 \pi}\left(\log \frac{\mu^{2}}{M^{2}}+1\right)-\frac{\alpha_{y}}{8 \pi}\right] .
$$

For the sake of comparison between the diagrammatic and the functional approach, we have split the result by diagram topology: the first term originates from the tree level graphs, the second one from the vertex graphs and the third one from the box diagrams. The propagator correction is scaleless and thus vanishes. In the functional computation, this result comes together in a somewhat different form. The supertraces yielding the vertex corrections are of the form:

$$
\operatorname{STr}\left\{\Delta_{\Psi} X_{\Psi \psi}^{[1]} \Delta_{\psi} X_{\psi \phi}^{[7 / 2]} \Delta_{\phi} X_{\phi \Psi}^{[3 / 2]}\right\}
$$


Note that this expression, once evaluated, involves the classical $\Psi$ through $X_{\psi \phi}^{[7 / 2]}$. To obtain the effective Lagrangian, this field has to be reduced by its equations of motion, turning the result into an expression of the form of $o_{6}$. Contributions corresponding to the third diagram in figure 2 never appear in the functional computation because the corresponding supertrace does not involve any heavy (quantum) fields. Finally, the box-type contributions are found directly from supertraces of the form:

$$
\operatorname{STr}\left\{\Delta_{\Psi} X_{\Psi \psi}^{[1]} \Delta_{\psi} X_{\psi \Psi}^{[1]} \Delta_{\Psi} X_{\Psi \phi}^{[3 / 2]} \Delta_{\phi} X_{\phi \Psi}^{[3 / 2]}\right\}
$$

The rest of the calculation proceeds analogously to the concepts explained here, and we refrain from detailing every step of the computation. Instead, we simply give the remaining matching coefficients,

$$
c_{\lambda}=24 \alpha_{y}^{2} \log \frac{\mu^{2}}{M^{2}}, \quad c_{7}=13 \alpha_{y}^{2}, \quad c_{8}=\frac{8}{3} \alpha_{y} \alpha, \quad c_{9}=\frac{15|y|^{6}}{\pi^{2}},
$$

which reproduces the output found from SuperTracer exactly.

Open Access. This article is distributed under the terms of the Creative Commons Attribution License (CC-BY 4.0), which permits any use, distribution and reproduction in any medium, provided the original author(s) and source are credited.

\section{References}

[1] W. Buchmüller and D. Wyler, Effective Lagrangian Analysis of New Interactions and Flavor Conservation, Nucl. Phys. B 268 (1986) 621 [INSPIRE].

[2] B. Grzadkowski, M. Iskrzynski, M. Misiak and J. Rosiek, Dimension-Six Terms in the Standard Model Lagrangian, JHEP 10 (2010) 085 [arXiv:1008.4884] [INSPIRE].

[3] E.E. Jenkins, A.V. Manohar and M. Trott, Renormalization Group Evolution of the Standard Model Dimension Six Operators I: Formalism and lambda Dependence, JHEP 10 (2013) 087 [arXiv: 1308.2627] [INSPIRE].

[4] E.E. Jenkins, A.V. Manohar and M. Trott, Renormalization Group Evolution of the Standard Model Dimension Six Operators II: Yukawa Dependence, JHEP 01 (2014) 035 [arXiv: 1310.4838] [INSPIRE].

[5] R. Alonso, E.E. Jenkins, A.V. Manohar and M. Trott, Renormalization Group Evolution of the Standard Model Dimension Six Operators III: Gauge Coupling Dependence and Phenomenology, JHEP 04 (2014) 159 [arXiv:1312.2014] [INSPIRE].

[6] R. Alonso, H.-M. Chang, E.E. Jenkins, A.V. Manohar and B. Shotwell, Renormalization group evolution of dimension-six baryon number violating operators, Phys. Lett. B 734 (2014) 302 [arXiv: 1405.0486] [INSPIRE].

[7] E.E. Jenkins, A.V. Manohar and P. Stoffer, Low-Energy Effective Field Theory below the Electroweak Scale: Operators and Matching, JHEP 03 (2018) 016 [arXiv:1709.04486] [INSPIRE].

[8] W. Dekens and P. Stoffer, Low-energy effective field theory below the electroweak scale: matching at one loop, JHEP 10 (2019) 197 [arXiv:1908.05295] [INSPIRE]. 
[9] J. Aebischer, A. Crivellin, M. Fael and C. Greub, Matching of gauge invariant dimension-six operators for $b \rightarrow s$ and $b \rightarrow c$ transitions, JHEP 05 (2016) 037 [arXiv: 1512.02830] [INSPIRE].

[10] E.E. Jenkins, A.V. Manohar and P. Stoffer, Low-Energy Effective Field Theory below the Electroweak Scale: Anomalous Dimensions, JHEP 01 (2018) 084 [arXiv:1711.05270] [INSPIRE].

[11] A. Celis, J. Fuentes-Martin, A. Vicente and J. Virto, DsixTools: The Standard Model Effective Field Theory Toolkit, Eur. Phys. J. C 77 (2017) 405 [arXiv:1704.04504] [InSPIRE].

[12] J. Aebischer, J. Kumar and D.M. Straub, Wilson: a Python package for the running and matching of Wilson coefficients above and below the electroweak scale, Eur. Phys. J. C 78 (2018) 1026 [arXiv: 1804.05033] [INSPIRE].

[13] J. Fuentes-Martin, P. Ruiz-Femenia, A. Vicente and J. Virto, DsixTools 2.0: The Effective Field Theory Toolkit, Eur. Phys. J. C 81 (2021) 167 [arXiv:2010.16341] [InSPIRE].

[14] J.C. Criado, MatchingTools: a Python library for symbolic effective field theory calculations, Comput. Phys. Commun. 227 (2018) 42 [arXiv:1710.06445] [INSPIRE].

[15] J. Aebischer, M. Fael, A. Lenz, M. Spannowsky and J. Virto eds., Computing Tools for the SMEFT, in proceedings of the 1st Workshop on Tools for Low-Energy SMEFT

Phenomenology (SMEFT-Tools 2019), Durham, U.K., 12-14 June 2019, arXiv:1910.11003 [INSPIRE].

[16] B. Gripaios and D. Sutherland, DEFT: A program for operators in EFT, JHEP 01 (2019) 128 [arXiv: 1807.07546] [INSPIRE].

[17] J.C. Criado, BasisGen: automatic generation of operator bases, Eur. Phys. J. C 79 (2019) 256 [arXiv: 1901.03501$]$ [INSPIRE].

[18] A. Dedes, M. Paraskevas, J. Rosiek, K. Suxho and L. Trifyllis, SmeftFR - Feynman rules generator for the Standard Model Effective Field Theory, Comput. Phys. Commun. 247 (2020) 106931 [arXiv: 1904.03204] [INSPIRE].

[19] N.P. Hartland et al., A Monte Carlo global analysis of the Standard Model Effective Field Theory: the top quark sector, JHEP 04 (2019) 100 [arXiv: 1901.05965] [INSPIRE].

[20] J. Aebischer, J. Kumar, P. Stangl and D.M. Straub, A Global Likelihood for Precision Constraints and Flavour Anomalies, Eur. Phys. J. C 79 (2019) 509 [arXiv:1810.07698] [INSPIRE].

[21] D. van Dyk et al., EOS HEP program for Flavor Observables, (2016) https://eos.github.io.

[22] D.M. Straub, flavio: a Python package for flavour and precision phenomenology in the Standard Model and beyond, arXiv:1810.08132 [INSPIRE].

[23] I. Brivio, Y. Jiang and M. Trott, The SMEFTsim package, theory and tools, JHEP 12 (2017) 070 [arXiv: 1709. 06492] [INSPIRE].

[24] G. Uhlrich, F. Mahmoudi and A. Arbey, MARTY - Modern ARtificial Theoretical phYsicist: A $C++$ framework automating symbolic calculations Beyond the Standard Model, Comput. Phys. Commun. 264 (2021) 107928 [arXiv:2011.02478] [INSPIRE].

[25] S. Das Bakshi, J. Chakrabortty and S.K. Patra, CoDEx: Wilson coefficient calculator connecting SMEFT to UV theory, Eur. Phys. J. C 79 (2019) 21 [arXiv:1808.04403] [INSPIRE]. 
[26] J. de Blas, J.C. Criado, M. Pérez-Victoria and J. Santiago, Effective description of general extensions of the Standard Model: the complete tree-level dictionary, JHEP 03 (2018) 109 [arXiv: 1711.10391] [INSPIRE].

[27] M. Krämer, B. Summ and A. Voigt, Completing the scalar and fermionic Universal One-Loop Effective Action, JHEP 01 (2020) 079 [arXiv:1908.04798] [INSPIRE].

[28] A. Angelescu and P. Huang, Integrating Out New Fermions at One Loop, JHEP 01 (2021) 049 [arXiv: 2006.16532] [inSPIRE].

[29] S.A.R. Ellis, J. Quevillon, P.N.H. Vuong, T. You and Z. Zhang, The Fermionic Universal One-Loop Effective Action, JHEP 11 (2020) 078 [arXiv:2006.16260] [INSPIRE].

[30] C. Anastasiou, A. Carmona, A. Lazopoulos and J. Santiago, in preparation.

[31] M.K. Gaillard, The Effective One Loop Lagrangian With Derivative Couplings, Nucl. Phys. B 268 (1986) 669 [INSPIRE].

[32] L.-H. Chan, Derivative Expansion for the One Loop Effective Actions With Internal Symmetry, Phys. Rev. Lett. 57 (1986) 1199 [inSPIRE].

[33] O. Cheyette, Effective Action for the Standard Model With Large Higgs Mass, Nucl. Phys. B 297 (1988) 183 [INSPIRE].

[34] L.-H. Chan, Effective Action Expansion in Perturbation Theory, Phys. Rev. Lett. 54 (1985) 1222 [Erratum ibid. 56 (1986) 404] [INSPIRE].

[35] C.M. Fraser, Calculation of Higher Derivative Terms in the One Loop Effective Lagrangian, Z. Phys. C 28 (1985) 101 [inSPIRE].

[36] I.J.R. Aitchison and C.M. Fraser, Fermion Loop Contribution to Skyrmion Stability, Phys. Lett. B 146 (1984) 63 [INSPIRE].

[37] I.J.R. Aitchison and C.M. Fraser, Derivative Expansions of Fermion Determinants: Anomaly Induced Vertices, Goldstone-Wilczek Currents and Skyrme Terms, Phys. Rev. D 31 (1985) 2605 [INSPIRE].

[38] I.J.R. Aitchison and C.M. Fraser, Trouble With Boson Loops in Skyrmion Physics, Phys. Rev. D 32 (1985) 2190 [INSPIRE].

[39] O. Cheyette, Derivative Expansion of the Effective Action, Phys. Rev. Lett. 55 (1985) 2394 [INSPIRE].

[40] S. Dittmaier and C. Grosse-Knetter, Deriving nondecoupling effects of heavy fields from the path integral: A Heavy Higgs field in an $\mathrm{SU}(2)$ gauge theory, Phys. Rev. D 52 (1995) 7276 [hep-ph/9501285] [INSPIRE].

[41] S. Dittmaier and C. Grosse-Knetter, Integrating out the standard Higgs field in the path integral, Nucl. Phys. B 459 (1996) 497 [hep-ph/9505266] [INSPIRE].

[42] B. Henning, X. Lu and H. Murayama, How to use the Standard Model effective field theory, JHEP 01 (2016) 023 [arXiv: 1412.1837] [InSPIRE].

[43] A. Drozd, J. Ellis, J. Quevillon and T. You, The Universal One-Loop Effective Action, JHEP 03 (2016) 180 [arXiv: 1512.03003] [INSPIRE].

[44] F. del Aguila, Z. Kunszt and J. Santiago, One-loop effective lagrangians after matching, Eur. Phys. J. C 76 (2016) 244 [arXiv:1602.00126] [INSPIRE]. 
[45] M. Boggia, R. Gomez-Ambrosio and G. Passarino, Low energy behaviour of standard model extensions, JHEP 05 (2016) 162 [arXiv: 1603.03660] [INSPIRE].

[46] B. Henning, X. Lu and H. Murayama, One-loop Matching and Running with Covariant Derivative Expansion, JHEP 01 (2018) 123 [arXiv:1604.01019] [INSPIRE].

[47] S.A.R. Ellis, J. Quevillon, T. You and Z. Zhang, Mixed heavy-light matching in the Universal One-Loop Effective Action, Phys. Lett. B 762 (2016) 166 [arXiv:1604.02445] [INSPIRE].

[48] J. Fuentes-Martin, J. Portoles and P. Ruiz-Femenia, Integrating out heavy particles with functional methods: a simplified framework, JHEP 09 (2016) 156 [arXiv: 1607.02142] [INSPIRE].

[49] Z. Zhang, Covariant diagrams for one-loop matching, JHEP 05 (2017) 152 [arXiv: 1610.00710] [INSPIRE].

[50] S.A.R. Ellis, J. Quevillon, T. You and Z. Zhang, Extending the Universal One-Loop Effective Action: Heavy-Light Coefficients, JHEP 08 (2017) 054 [arXiv:1706. 07765] [INSPIRE].

[51] B. Summ and A. Voigt, Extending the Universal One-Loop Effective Action by Regularization Scheme Translating Operators, JHEP 08 (2018) 026 [arXiv: 1806.05171] [INSPIRE].

[52] T. Cohen, M. Freytsis and X. Lu, Functional Methods for Heavy Quark Effective Theory, JHEP 06 (2020) 164 [arXiv: 1912.08814] [INSPIRE].

[53] T. Cohen, X. Lu and Z. Zhang, Functional Prescription for EFT Matching, JHEP 02 (2021) 228 [arXiv: 2011.02484] [INSPIRE].

[54] T. Cohen, X. Lu and Z. Zhang, STrEAMlining EFT Matching, arXiv:2012.07851 [INSPIRE].

[55] M. Beneke and V.A. Smirnov, Asymptotic expansion of Feynman integrals near threshold, Nucl. Phys. B 522 (1998) 321 [hep-ph/9711391] [INSPIRE].

[56] B. Jantzen, Foundation and generalization of the expansion by regions, JHEP 12 (2011) 076 [arXiv:1111.2589] [INSPIRE].

[57] R.D. Ball, Chiral Gauge Theory, Phys. Rept. 182 (1989) 1 [INSPIRE].

[58] F. Jegerlehner, Facts of life with gamma(5), Eur. Phys. J. C 18 (2001) 673 [hep-th/0005255] [INSPIRE].

[59] M.S. Chanowitz, M. Furman and I. Hinchliffe, The Axial Current in Dimensional Regularization, Nucl. Phys. B 159 (1979) 225 [INSPIRE].

[60] L.N. Mihaila, J. Salomon and M. Steinhauser, Renormalization constants and $\beta$-functions for the gauge couplings of the Standard Model to three-loop order, Phys. Rev. D 86 (2012) 096008 [arXiv: 1208.3357 ] [INSPIRE].

[61] J. Fuentes-Martin, M. König, J. Pagès, A.E. Thomsen and F. Wilsch, in preparation.

[62] V. Gherardi, D. Marzocca and E. Venturini, Matching scalar leptoquarks to the SMEFT at one loop, JHEP 07 (2020) 225 [Erratum JHEP 01 (2021) 006] [arXiv:2003.12525] [INSPIRE]. 